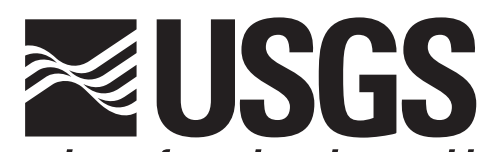

science for a changing world

\title{
USGS Polar Temperature Logging System, Description and M easurement Uncertainties
}

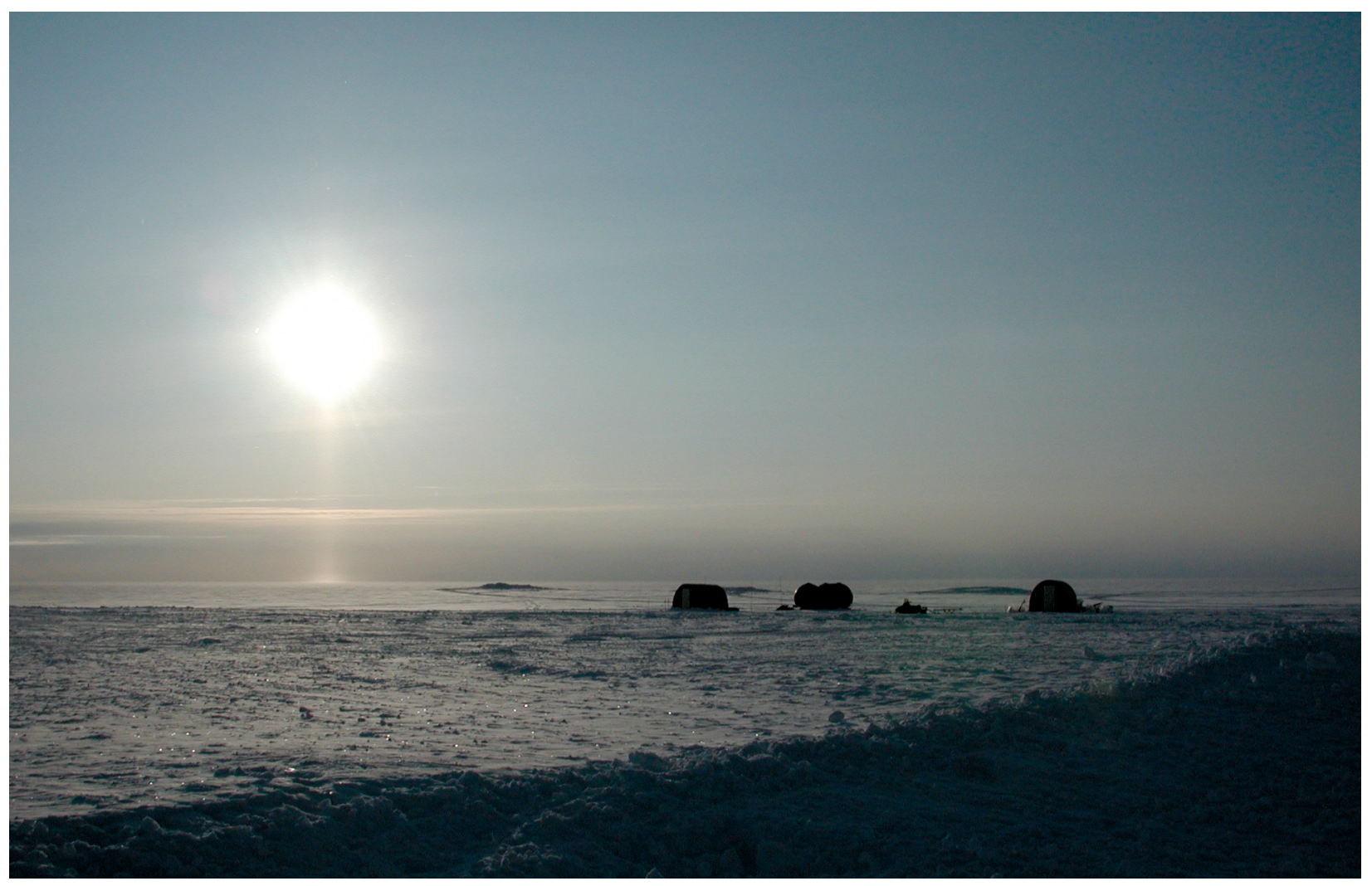

Techniques and M ethods 2-E3

U.S. Department of the Interior U.S. Geological Survey 
Cover photograph: North Greenland Ice Core Project ic e-coring site in central Greenland. (Photograph by Gary D. Clow, U.S. Geological Survey) 


\section{USGS Polar Temperature Logging System, Description and Measurement Uncertainties}

By Gary D. Clow

Techniques and Methods 2-E3 


\section{U.S. Department of the Interior DIRK KEMPTHORNE, Secretary}

\section{U.S. Geological Survey \\ Mark D. Myers, Director}

\section{U.S. Geological Survey, Reston, Virginia: 2008}

For product and ordering information:

World Wide Web: http://www.usgs.gov/pubprod

Telephone: 1-888-ASK-USGS

For more information on the USGS —-the Federal source for science about the Earth, its natural and living resources, natural hazards, and the environment:

World Wide Web: http://www.usgs.gov

Telephone: 1-888-ASK-USGS

Any use of trade, product, or firm names is for descriptive purposes only and does not imply endorsement by the U.S. Government.

Although this report is in the public domain, permission must be secured from the individual copyright owners to reproduce any copyrighted materials contained within this report.

Suggested citation:

Clow, Gary D., 2008, USGS Polar Temperature Logging System, Description and Measurement Uncertainties: U.S. Geological Survey Techniques and Methods 2 -E3, 24 p.

Available online at: http://pubs.usgs.gov/tm/02e03 


\section{Contents}

$\begin{array}{lr}\text { Abstract } & 1\end{array}$

1 Introduction $\quad 1$

2 System Description $\quad 2$

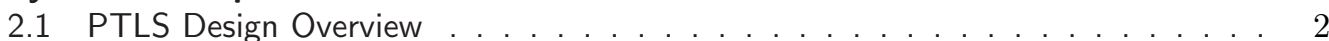

2.2 Resistance (Temperature) Measurement System . . . . . . . . . . . . . . . . . 3

2.2 .1 Kelvin Circuit . . . . . . . . . . . . . . . . . . . . 3

2.2.2 Resistance Corrections ...................... 4

2.2 .3 Noise . . . . . . . . . . . . . . . . . . . . . 5

2.2.4 Temperature Sensors . . . . . . . . . . . . . . . . . . . . . . . . . . . . 7

2.3 Depth-Measurement System . . . . . . . . . . . . . . . . . . . . . . . . . . 8

2.3.1 System Description . . . . . . . . . . . . . . . . . . 8

2.3.2 Depth Corrections . . . . . . . . . . . . . . . . . . . 9

3 Measurement Uncertainties $\quad 11$

3.1 ITS-90 Temperature Uncertainties . . . . . . . . . . . . . . . . . . . 11

3.1.1 Resistance Readout Uncertainties . . . . . . . . . . . . . . . . . . . . . 11

3.1.2 Kelvin Circuit Uncertainties . . . . . . . . . . . . . . . . . . . . 12

3.1.3 Temperature-Sensor Calibration Uncertainties . . . . . . . . . . . . . . . . . 13

3.1.4 Combined ITS-90 Temperature Uncertainties . . . . . . . . . . . . . . . 16

3.2 Relative Temperature Uncertainties . . . . . . . . . . . . . . . . . . . . . . 17

3.3 Depth Uncertainties . . . . . . . . . . . . . . . . . . . . . . . 18

3.4 Example: Proposed WAIS Divide Borehole, Antarctica . . . . . . . . . . . . . . 20

4 Summary $\quad 22$

$\begin{array}{ll}\text { Appendix: Nomenclature } & 23\end{array}$

$\begin{array}{lr}\text { References } & 24\end{array}$ 


\section{Figures}

Primary PTLS components . . . . . . . . . . . . . . . . . . . . . . 3

2 Kelvin resistance circuit . . . . . . . . . . . . . . . . . . . . . 4

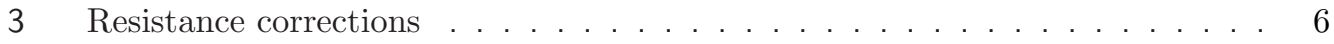

4 Raw noise, example. . . . . . . . . . . . . . . . . . . . . 7

5 Temperature-sensor design . . . . . . . . . . . . . . . . . . . . . . 8

$6 \quad \alpha_{T}$ values for various probe series . . . . . . . . . . . . . . . . . . . 8

7 Temperature-sensor calibration data, example . . . . . . . . . . . . . . . . . 8

8 Temperature resolution . . . . . . . . . . . . . . . . . . . . . . . . 9

9 Force-dependent function $\mathcal{F}(\tilde{Z})$, example . . . . . . . . . . . . . . . . . . . . 10

10 Resistance correction uncertainties . . . . . . . . . . . . . . . . . . . 14

11 Temperature-sensor calibration uncertainties . . . . . . . . . . . . . 15

12 Damping of calibration bath's temperature fluctuations . . . . . . . . . . . 16

13 Combined ITS-90 temperature measurement uncertainty . . . . . . . . . . . . 17

14 Combined relative temperature measurement uncertainty . . . . . . . . . . . 18

15 Example: Temperature uncertainties, WAIS Divide borehole . . . . . . . . . . 20

16 Example: Depth uncertainties, WAIS Divide borehole . . . . . . . . . . . 21

\section{Tables}

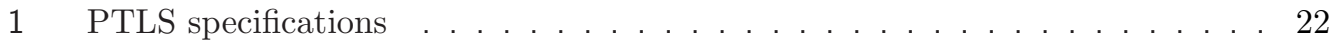




\title{
USGS Polar Temperature Logging System, Description and Measurement Uncertainties
}

\author{
By Gary D. Clow
}

\begin{abstract}
This paper provides an updated technical description of the USGS Polar Temperature Logging System (PTLS) and a complete assessment of the measurement uncertainties. This measurement system is used to acquire subsurface temperature data for climate-change detection in the polar regions and for reconstructing past climate changes using the "borehole paleothermometry" inverse method. Specifically designed for polar conditions, the PTLS can measure temperatures as low as $-60^{\circ}$ Celsius with a sensitivity ranging from 0.02 to 0.19 millikelvin $(\mathrm{mK})$. A modular design allows the PTLS to reach depths as great as 4.5 kilometers with a skid-mounted winch unit or 650 meters with a small helicopter-transportable unit. The standard uncertainty $\left(u_{T}\right)$ of the ITS-90 temperature measurements obtained with the current PTLS range from $3.0 \mathrm{mK}$ at $-60^{\circ}$ Celsius to $3.3 \mathrm{mK}$ at $0^{\circ}$ Celsius. Relative temperature measurements used for borehole paleothermometry have a standard uncertainty $\left(u_{T}^{r}\right)$ whose upper limit ranges from $1.6 \mathrm{mK}$ at $-60^{\circ}$ Celsius to $2.0 \mathrm{mK}$ at $0^{\circ}$ Celsius. The uncertainty of a temperature sensor's depth during a log depends on specific borehole conditions and the temperature near the winch and thus must be treated on a case-by-case basis. However, recent experience indicates that when logging conditions are favorable, the 4.5-kilometer system is capable of producing depths with a standard uncertainty $\left(u_{Z}\right)$ on the order of 200-250 parts per million.
\end{abstract}

\section{Introduction}

In 1993, the U.S. Geological Survey (USGS) began developing a new borehole temperature logging system specifically to address emerging climate issues in the polar regions. This system, referred to as the USGS Polar Temperature Logging System (PTLS), has two primary functions. (1) Periodically obtain subsurface temperature data from arrays of polar boreholes for climate-change detection. Monitoring data acquired by the PTLS in northern Alaska contributes to the Global Climate Observing System (GCOS) through the Global Terrestrial Network for Permafrost (GTNP). (2) Acquire data for the reconstruction of past climate changes in the polar regions using "borehole paleothermometry." Climate reconstructions derived from borehole temperature measurements are hindered by considerable temporal smearing due to heat diffusion processes. While it cannot be eliminated, the extent of the temporal averaging can be minimized through optimal experimental design. Application of Backus-Gilbert inverse methods to the paleoclimate reconstruction problem shows that our ability to resolve past climatic events can be optimized by reducing the uncertainty in the temperature measurements to no more than 0.1 percent of the paleoclimate signal we are attempting to detect (Clow, 1992). In most places on Earth, surface-temperature changes during the Holocene were on the order of $\pm 1 \mathrm{~K}$. Thus to enhance our ability to resolve past climate events of this magnitude, it is desirable to reduce the uncertainty of the borehole temperature measurements to about $1 \mathrm{mK}$. Paleoclimate reconstruction has been the primary driver for the design requirements of the PTLS since its requirements are much more stringent than those needed for detecting contemporary climate change.

The objectives of this paper are to provide an updated technical description of the PTLS logging system and an analysis of the measurement uncertainties. This system and its uncertainties were originally described by Clow and others (1996). However, the PTLS is a continually evolving system, warranting an updated description. In addition, calibration facilities and procedures have changed significantly since 1996. The current paper provides a much more complete analysis 


\section{$2 \quad$ USGS Polar Temperature Logging System}

of the measurement uncertainties than was possible in Clow and others (1996). The need for such an analysis is twofold: (1) The usefulness of scientific data produced by monitoring systems critically depends on the availability of thorough uncertainty analyses. This is particularly true of climate-monitoring systems. (2) To reconstruct past climate changes using borehole paleothermometry, one needs to know the uncertainties of the data. This requirement, shared by all geophysical inverse techniques (Parker, 1994), determines the very structure of the derived climate histories. In conformance with ISO standards (ISO, 1993a; ISO, 1993b), we use the CIPM ${ }^{1}$ approach for expressing and evaluating the measurement uncertainties of the PTLS.

\section{System Description}

\subsection{PTLS Design Overview}

A variety of system designs presently (2008) are used to measure temperatures in geophysical boreholes. Most systems use either a temperature-dependent resistive element (thermistor or RTD) or a piezoelectric crystal whose resonant frequency is temperaturesensitive for the sensing element. In the former case, the resistance of the sensing element is measured by a custom-built electronic bridge or by a commercial resistance readout. An advantage of a custom bridge is that it can be made small enough to be included in an instrument package located at the downhole end of the logging cable, keeping the electronic lead lengths to the sensor relatively short. Such a package can be designed to measure several other parameters as well, such as fluid pressure and borehole inclination, and the resulting data either stored within the instrument package or digitally transmitted to the surface. A significant disadvantage of this approach for precision thermometry is the difficulty of maintaining the calibration of the electronic bridge while the instrument package experiences temperature changes of 10-30 K during the course of a logging experiment. The associated calibration drift of the bridge can produce temperature measurement errors of $10 \mathrm{mK}$, or more. Application of new technologies may substantially reduce these errors, making high-precision downhole digital thermometers possible in the near future. The current alternative is to locate the resistance-measuring circuitry on the surface. The advantages of this strategy are: (1) a high-quality commercial resistance readout can be

\footnotetext{
${ }^{1}$ International Committee for Weights and Measures.
}

utilized for the measuring circuitry instead of having to develop custom miniaturized circuits, (2) the readout can be maintained at a constant temperature, eliminating temperature-related drift in the measurement circuit, and (3) the calibration of the readout can be periodically rechecked while measurements are in progress. However, the long lead lengths between the resistance readout and the sensor, potentially up to $10 \mathrm{~km}$, make the measurements vulnerable to several sources of instrumental error. Great care must be exercised to minimize these errors. Logging systems with a downhole electronic bridge generally acquire temperature measurements while lowering the sensor downhole at a constant speed ("continuous" logging). Another common technique is to acquire data with the probe stopped at a fixed depth; repeating this process at multiple depths yields an incremental or "stop-andgo" temperature log. Systems with the resistancemeasuring circuitry on the surface are sometimes limited to this mode. Although incremental logging yields measurements at a limited number of depths, these measurements are free of the "slip-ring" noise (see Section 2.2) inherent to continuous temperature logs obtained with surface measurement circuitry.

Considering the advantages and disadvantages of various system designs along with our scientific objectives, the following requirements were established for the Polar Temperature Logging System: the system must be modular and flexible so it can use a variety of sensors, can make measurements using either downhole or surface measurement circuitry, can be operated in either the continuous or incremental logging modes, and can utilize different length logging cables depending on borehole depth and available logistics. In addition, the system must be able to measure temperatures as low as $-60^{\circ} \mathrm{C}$ with an uncertainty of about $1 \mathrm{mK}$, reach depths comparable to the maximum thickness of the polar ice sheets (4$5 \mathrm{~km}$ ), work in the presence of strong environmental noise (for example, changing electrostatic fields), and be rugged enough to survive offloading from military cargo aircraft. The PTLS evolved from a number of refinements to a conventional temperature logging system design, thereby avoiding the need to build a radically new system. This approach took advantage of the USGS' considerable experience in borehole thermometry. Negative-temperature-coefficient (NTC) hermetically sealed thermistors were selected for the primary temperature sensors because of their ruggedness, stability, and high temperature coefficient, which helps produce a high system sensitivity. A 


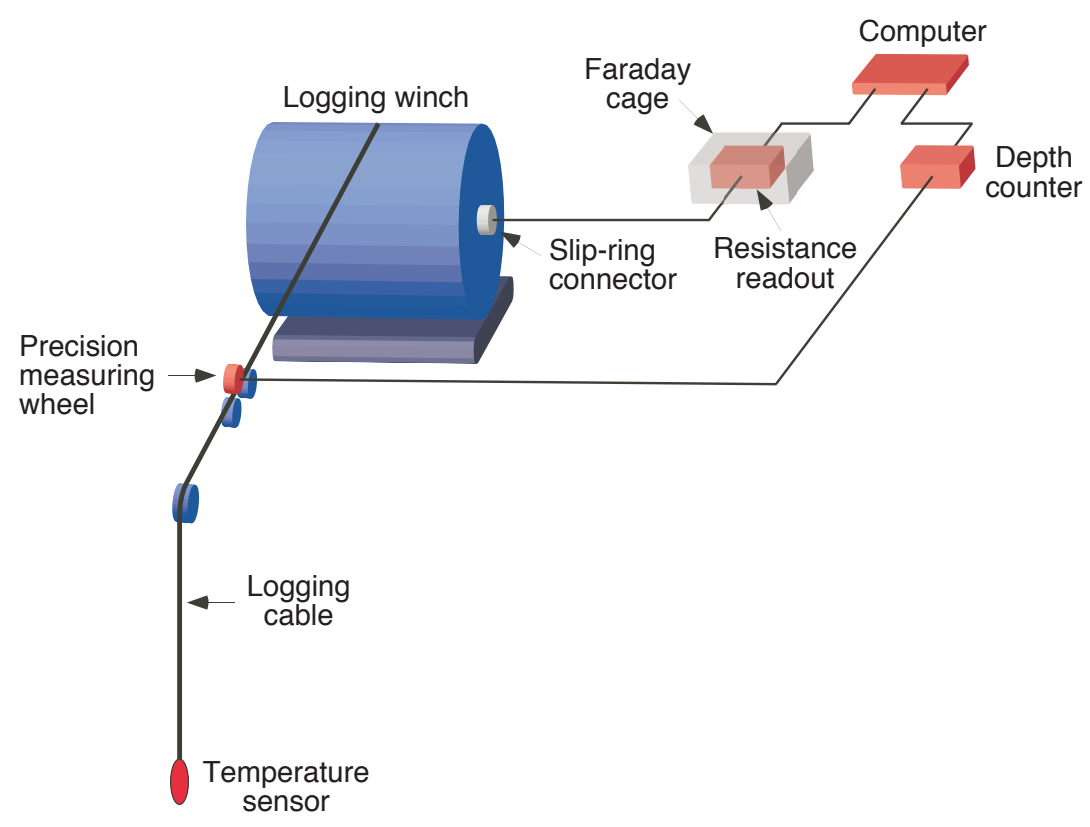

Figure 1. Layout of the USGS Polar Temperature Logging System when using surface measurement circuitry.

commercial resistance readout located on the surface is normally used for the resistance-measuring circuitry. This circuitry is suspended inside a Faraday cage maintained at $23 \pm 0.5^{\circ} \mathrm{C}$ for the duration of an experiment; thermal stability is provided by microprocessorcontrolled etched-foil heaters. As an alternative to surface measurement circuitry, a prototype downhole digital thermometer utilizing NTC thermistors has been used with the PTLS. However, this device is still in the testing phase and will not be discussed further here. Other major system components include a 4conductor logging cable mounted on a motorized winch (fig. 1). Two different size winches are currently available: a small helicopter-transportable unit with $650 \mathrm{~m}$ of cable, and a much larger skid-mounted unit capable of reaching $4.5 \mathrm{~km}$. A "slip-ring" assembly provides electrical continuity between the logging cable and the surface electronics. Depth information is provided by an optical encoder mounted on a calibrated measuring wheel. A laptop computer controls the system, both displaying and storing the measured resistance, depth, time, and logging speed. Cable tension provided by a strain-gage force transducer is displayed on a separate monitor. To minimize electrical noise, all components are powered by DC batteries except for the winch motors. The Faraday cage surrounding the resistance readout, used in conjunction with cable shielding, helps isolate the measurement circuitry from the remaining sources of electrical noise.

The three fundamental measurements made by the PTLS are the sensor resistance, sensor depth, and time of data acquisition. For the time measurements, we simply rely on the computer's onboard clock. The resistance and depth measurements are more involved and are described in detail in the following sections. Resistance measurements are subsequently converted to temperature using a 4-term conversion function.

\subsection{Resistance (Temperature) Measurement System}

\subsubsection{Kelvin Circuit}

To measure the resistance, a Kelvin (4-wire) circuit is used in both the downhole and the surface measurement-circuitry configurations (fig. 2). During this measurement, the resistance readout produces a highly regulated current $\left(I_{s}\right)$ that passes through the sensor. The resulting voltage drop $(\Delta V)$ across the probe is detected by the readout's high-impedance inputs, and the probe's resistance calculated from $\tilde{R}_{s}=\Delta V / I_{s}$. This 4-wire measurement eliminates the effect of the lead resistance $\left(R_{L}\right)$ along each conductor between the resistance readout and the temperature sensor. There are, however, several sources of systematic error that potentially require correction. 
USGS Polar Temperature Logging System

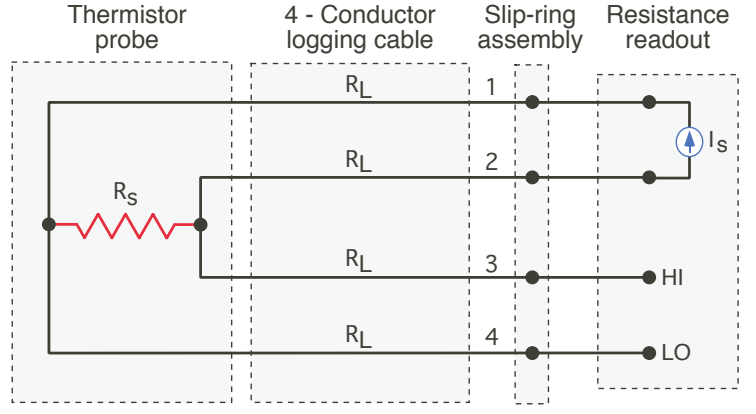

Figure 2. Kelvin (4-wire) resistance circuit used by the PTLS when the measurement circuitry is located on the surface. The test current $I_{s}$ passes through lines 1 and 2 while the voltage drop across $R_{s}$ is measured using the sense lines $(3,4)$. With downhole circuitry, an electronic bridge is connected directly to the probe.

\subsubsection{Resistance Corrections}

Leakage paths between the conductors of the Kelvin circuit can produce significant systematic error, particularly when the probe resistance $R_{s}$ is large. Such unintended paths arise due to leakage currents passing directly through the conductor insulation, moisture absorption by the insulation, or leakage due to contaminants on the surface of the insulation or connectors. Leakage currents between the circuit's sense lines or between the current-carrying lines will reduce the measured resistance by

$$
\delta R_{l}=\frac{R_{s}^{2}}{R_{s}+R_{l}}
$$

where $R_{l}$ is the leakage resistance. Teflon is used for the Kelvin circuit insulation because of its high volume resistivity (greater than $10^{16} \Omega \cdot \mathrm{m}$ ), low water absorption, and a surface that tends to repel many films. This problem is further controlled by rigorously cleaning the connectors and by using a sensor with a low resistance $R_{s}$. Part of our logging protocol is to measure the resistance $R_{l}$ between each pair of conductors immediately before each logging experiment. If $R_{l}$ is less than $10 \mathrm{G} \Omega$ for any pair of conductors, the $\log$ is aborted until the problem is rectified. $R_{l}$ is greater than $20 \mathrm{G} \Omega$ for nearly all logging experiments conducted with the PTLS.

A capacitor is occasionally introduced between the readout's sense lines to perform high-frequency noise filtering. This also delays the response of the circuit so that the measured resistance is too low by

$$
\delta R_{c}=\tau \frac{\partial R_{s}}{\partial t} .
$$

$\tau=R_{s} C$ is the circuit's natural response time where $C$ is the capacitance. If a filtering capacitor is used, the resistance offset $\delta R_{c}$ is controlled by keeping $\tau$ small relative to the measurement integration time of the resistance readout and by using a slow logging speed $v$ so that the rate of resistance change $\partial R_{s} / \partial t=$ $v \alpha_{T} R_{s}(\partial T / \partial z)$ is also small; $\alpha_{T}$ is the sensor's temperature coefficient of resistance $\left(\alpha_{T} \equiv R_{s}^{-1} \partial R_{s} / \partial T\right)$. With typical logging speeds $\left(2.5-5.5 \mathrm{~cm} \cdot \mathrm{s}^{-1}\right)$ and temperature gradients $\left(\partial T / \partial z \leq 50 \mathrm{mK} \cdot \mathrm{m}^{-1}\right)$, the most rapid resistance changes are on the order of $10^{-4} R_{s}$ per second. Without the filtering capacitor, $C$ is determined by the capacitance of the logging cable $(76 \mathrm{nF}$ for the $650-\mathrm{m}$ "short" cables and $0.68 \mu \mathrm{F}$ for the $4,600-$ m "long" cable).

As the current $I_{s}$ passes through the temperature sensor, power dissipates within the probe at a rate $P=$ $I_{s}^{2} R_{s}$. This warms the probe, effectively reducing its resistance by

$$
\delta R_{h}=\frac{\alpha_{T}\left(I_{s} R_{s}\right)^{2}}{P_{d}}
$$

where $P_{d}$ is the probe's power dissipation constant. The best strategy for minimizing self-heating is to use a resistance readout with a small source current $I_{s}$ and a probe with a relatively low resistance. The source current for the resistance readout used with the PTLS is $\leq 10 \mu \mathrm{A}$.

To provide modularity, the Kelvin circuit consists of a number of components attached to one another using high-quality electrical connectors (fig. 2). Different portions of the circuit operate at vastly different temperatures with the sensor-end of the circuit often being $30-70 \mathrm{~K}$ colder than the resistance readout. This situation has the potential to generate significant thermoelectric voltages (thermal EMFs) between electrical junctions separating dissimilar metals through the Seebeck effect (McGee, 1988). The sum of the thermal EMFs can be found by integrating the Seebeck coefficient $Q$ around the entire circuit, starting and ending at the resistance readout's $\mathrm{HI}$ and $\mathrm{LO}$ sense connections (junctions $a_{1}$ and $a_{2}$ ),

$$
V_{\mathrm{emf}}=-\int_{a_{1}}^{a_{2}} Q(T) d T .
$$

$Q$ depends primarily on metal composition and secondarily on temperature. If the metal compositions on the HI side of the circuit exactly match those on the LO side, the total thermal EMF can be expressed by 


$$
\begin{gathered}
V_{\mathrm{emf}}=-\int_{a_{1}}^{b_{1}} Q_{A}(T) d T-\int_{b_{1}}^{c_{1}} Q_{B}(T) d T-\cdots \\
-\int_{c_{2}}^{b_{2}} Q_{B}(T) d T-\int_{b_{2}}^{a_{2}} Q_{A}(T) d T
\end{gathered}
$$

where subscripts 1 and 2 refer to the HI and LO sides of the circuit, respectively; $Q_{A}$ is the Seebeck coefficient for the metal conductor between junctions $a_{1}$ and $b_{1}$ (and between $a_{2}$ and $b_{2}$ ), $Q_{B}$ is the coefficient for the metal between junctions $b_{1}$ and $c_{1}$, and so forth. Equation (5) can be rewritten in terms of the adjacent junction pairs on the $\mathrm{HI}$ and LO sides of the circuit (for example, $a_{1}$ and $a_{2}$ ),

$$
\begin{aligned}
V_{\mathrm{emf}} & =\left[\int_{a_{2}}^{a_{1}} Q_{A}(T) d T+\int_{b_{1}}^{b_{2}} Q_{A}(T) d T\right] \\
+ & {\left[\int_{b_{2}}^{b_{1}} Q_{B}(T) d T+\int_{c_{1}}^{c_{2}} Q_{B}(T) d T\right]+\cdots . }
\end{aligned}
$$

If the temperature difference between adjacent junction pairs is small enough that the temperature dependence of the Seebeck coefficient can be ignored, equation (6) reduces to

$$
\begin{aligned}
V_{\mathrm{emf}}=- & Q_{A}\left(T_{a_{2}}-T_{a_{1}}\right)+\left(Q_{A}-Q_{B}\right)\left(T_{b_{2}}-T_{b_{1}}\right) \\
& +\left(Q_{B}-Q_{C}\right)\left(T_{c_{2}}-T_{c_{1}}\right)+\cdots
\end{aligned}
$$

Thus, thermal EMFs can be controlled by minimizing the temperature difference between adjacent junction pairs. Based on this analysis, we use a number of strategies to minimize thermal EMFs in the PTLS' Kelvin circuit: (1) The use of dissimilar metals is kept to a minimum. Except for the junctions themselves, the circuit paths consist almost entirely of copper or silver-plated copper. (2) Where a dissimilar metal occurs on the HI side of the circuit, the metal is matched with an identical metal at the corresponding location on the LO side. (3) The temperature difference between adjacent junction pairs is minimized. This is accomplished by locating the junctions of an adjacent pair as close together as possible, locating junction pairs within the thermally controlled Faraday cage where thermal gradients are very small, and (or) by locating junction pairs within high-conductivity metal shells where temperature gradients are also small. Before every experiment, the resistance readout is warmed up for at least an hour to minimize thermal EMFs within the readout itself. Considering the estimated temperature differences at the adjacent junction pairs and the values of the Seebeck coefficients, the total thermal EMF for the Kelvin circuit is estimated to be on the order of $0.5 \mu \mathrm{V}$ with the dominant sources occurring at the slip-ring assembly. Current-reversal experiments with both the $650-\mathrm{m}$ logging cables and the 4,600-m cable confirm that $V_{\text {emf }}$ is typically $\leq 0.5 \mu \mathrm{V}$. This voltage offset increases the measured resistance by

$$
\delta R_{e}=\frac{V_{\mathrm{emf}}}{I_{s}} .
$$

In 2008, the simple constant-current source that the PTLS had used was changed to a reversing source. This allowed us to switch to a current-reversal technique where a "resistance measurement" is found by averaging two measurements made with currents of opposite polarity. With this approach, the thermal EMFs produced during each polarity completely cancel out so that $\delta R_{e}=0$.

The first three systematic errors $\left(\delta R_{l}, \delta R_{c}, \delta R_{h}\right)$ can be controlled by using a sensor with a relatively small resistance. However, an additional constraint imposed by the resistance readout is that $R_{s}$ must be much greater than the lead resistance $R_{L}$ for each leg of the Kelvin circuit in order to make an accurate resistance measurement. At a minimum, $R_{s}$ should be at least 20 times $R_{L}$. Other factors that help control the systematic errors are a small capacitance $C$, a slow logging speed $v$, a small source current $I_{s}$, carefully matching the composition of the wires, and keeping the temperature difference between adjacent junction pairs as small as possible. Despite efforts to control leakage paths, the capacitance effect, selfheating, and thermal EMFs, small systematic errors will remain. We attempt to eliminate these errors by applying equations (1) - (3) and (8) as corrections to the resistance $\tilde{R}_{s}$ recorded by the resistance readout to obtain our estimate of the temperature sensor's true resistance,

$$
R_{s}=\tilde{R}_{s}+\left(\delta R_{l}+\delta R_{c}+\delta R_{h}-\delta R_{e}\right) .
$$

Figure 3 shows the magnitude of the resistance corrections for the PTLS under typical operating conditions. Expressed in terms of temperature, the corrections are generally limited to $0.1-0.2 \mathrm{mK}$. The uncertainty of these systematic error corrections is discussed in Section 3.1.2.

\subsubsection{Noise}

Several sources of noise also perturb the resistance measurements when the measurement circuitry is lo- 


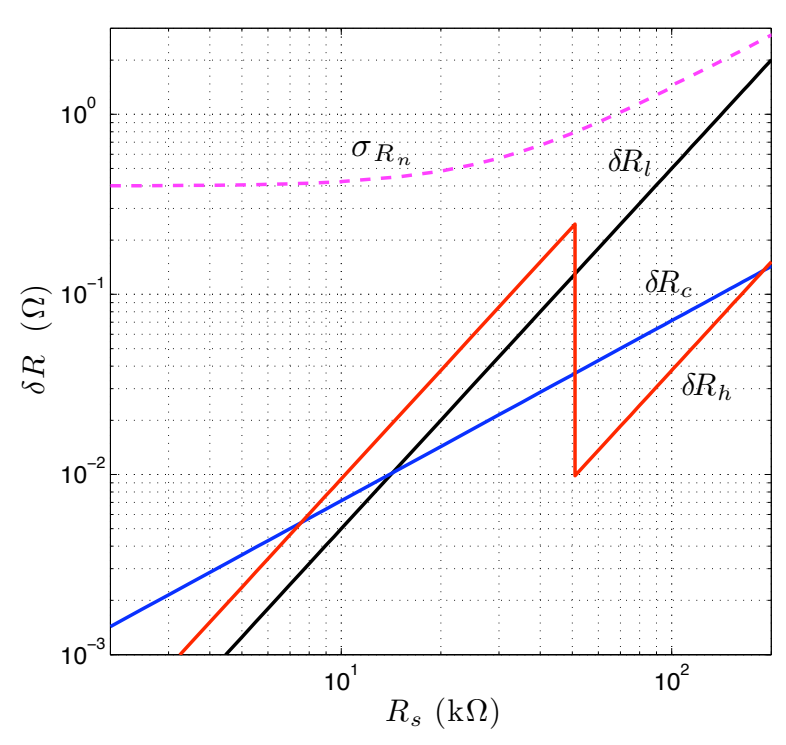

Figure 3. Magnitude of the resistance corrections $\delta R_{l}, \delta R_{c}, \delta R_{h}$ under typical operating conditions; $\delta R_{e}$ is essentially zero with the current PTLS design. In this example, the capacitance correction $\delta R_{c}$ assumes a logging speed of 5.5 centimeters per second, a temperature gradient of 25 millikelvins per meter, and a capacitance $C$ such that $\tau=10$ milliseconds. Equivalent temperature corrections are given by $\Delta T_{\mathrm{x}}=\delta R_{\mathrm{x}} /\left(\alpha_{T} R_{s}\right)$. Also shown is the approximate standard deviation $\sigma_{R_{n}}$ of the raw noise (eq. 10) generated in the Kelvin circuit under typical conditions.

cated on the surface. These sources include electrostatic coupling, electromagnetic EMFs, triboelectric effects, and switching effects. Depending on the source, the noise generated within the circuit consists either of extraneous voltages $V^{\prime}$ or extraneous currents $I^{\prime}$. The resistance readout internally converts the extraneous voltages into an apparent resistance noise, $R^{\prime}=$ $\left(V^{\prime} / I_{s}\right)$, while the extraneous currents are converted to $R^{\prime}=R_{s}\left(I^{\prime} / I_{s}\right)$. In the latter case, the apparent resistance noise increases in direct proportion to the temperature sensor's resistance.

Individual noise components: Electrostatic coupling occurs when an electrically charged object such as the system operator, moves near the Kelvin circuit, generating currents in the conductors. In polar environments, significant electrostatic interference can also be caused by electric charges transferred to the sheath of the logging cable by dry, blowing snow. Electromagnetic voltages (EMFs) are generated when a changing magnetic field passes through the conductive loop represented by the Kelvin circuit, or when some portion of the conductive loop (for example, the logging cable) moves relative to a magnetic field. For the PTLS, nearby AC fields, winch motors, and the Earth's magnetic field are potential sources of electromagnetic voltages. We use several strategies to mitigate the noise generated by electrostatic coupling and electromagnetic EMFs:

(1) Once a temperature log is initiated, the system runs in an automated mode, allowing the system operator to remain an adequate distance from the Kelvin circuit.

(2) The entire system is shielded from the wind and blowing snow as much as possible by operating it inside an insulated shelter. The Faraday cage and associated electronics are always operated within a protective shelter. If the wellhead cannot also be located inside the shelter, the shelter is placed as close as possible to the wellhead to minimize the amount of exposed logging cable.

(3) To minimize AC power fields, all system components are powered by DC batteries except for the winch motors.

(4) The most sensitive portions of the Kelvin circuit, especially the resistance readout, are kept as far as possible from the winch motors.

(5) All movement around the Kelvin circuit is kept to a minimum during a temperature log.

(6) The loop area of the circuit is minimized by using twisted-wire cables.

(7) The resistance readout is electrically shielded inside a Faraday cage. All the cables are also electrically shielded.

Given these noise-reduction strategies, the dominant sources of noise for the PTLS are believed to be due to switching effects within the electromechanical "slip-ring" assembly and to the triboelectric effect. Triboelectric currents arise from charges generated between the insulation and conductors of the logging cable as it flexes over the sheave wheels that guide it into a borehole. Triboelectric currents can also occur when the logging cable vibrates in the wind. We manage the triboelectric currents by providing a smooth path into the borehole, logging downhole at a slow steady pace with negligible accelerations, and by keeping the logging cable shielded from strong winds as much as possible. The slip-ring noise is controlled by using high-quality slip-rings. 
The internal noise of the resistance readout also contributes a small amount of noise to the recorded resistances, as does the truncation error associated with the instrument's finite resistance resolution $\Delta R_{r}$. Combining the readout noise with that due to the extraneous voltages and currents generated within the Kelvin circuit, the standard deviation of the noise in the resistance measurements is given by

$$
\sigma_{R_{n}}=\left[\left(a \Delta R_{r}\right)^{2}+\left(\frac{V^{\prime}}{I_{s}}\right)^{2}+\left(\frac{R_{s} I^{\prime}}{I_{s}}\right)^{2}\right]^{1 / 2} .
$$

Although the noise varies between temperature logs, depending on circumstances, the constants in equation (10) are generally on the order of $a \approx 0.33$, $V^{\prime} \approx 4 \mu \mathrm{V}$, and $I^{\prime} \approx 0.14 \mathrm{nA}$ for the PTLS under most conditions. Extraneous voltages dominate the noise for probe resistances less than $20 \mathrm{k} \Omega$ while extraneous currents dominate when $R_{s}>40 \mathrm{k} \Omega$ (fig. 3); the readout's contribution is relatively small at all resistances. The raw noise described by equation (10) can be substantially reduced by judiciously applying the resistance readout's internal filters or by installing a filtering capacitor between the circuit's sense lines. When these hardware noise filters are used, the noise $\sigma_{\tilde{R}_{n}}$ actually present in the recorded resistances is less than $\sigma_{R_{n}}$. The remaining noise is largely removed during data processing by using wavelet denoising techniques.

\subsubsection{Temperature Sensors}

Equations (1) - (3) show that the temperature sensor is an important factor in determining the characteristics of the overall measurement system. The primary sensors used with the PTLS consist of a parallel-series network of 15 negative-temperature-coefficient (NTC) thermistors divided into three packets. ${ }^{2}$ Each packet is hermetically sealed in glass (fig. 5) to prevent changes in the oxidation state of the metal oxide thermistors and to relieve strain where the leads are attached to the ceramic body of the thermistors. As a result, the probes have good long-term stability with typical drift rates of $\leq 0.025$ percent per year. The packets are wired in parallel so that only one third of the resistance readout's excitation current $I_{s}$ passes through any given thermistor bead, minimizing the self-heating effect. To improve the ruggedness of the design, the

\footnotetext{
${ }^{2}$ A similar design appropriate for temperatures at midlatitudes is described by Sass and others (1971). The midlatitude version has a much higher resistance than the polar model and contains 20 thermistors divided into two packets.
}

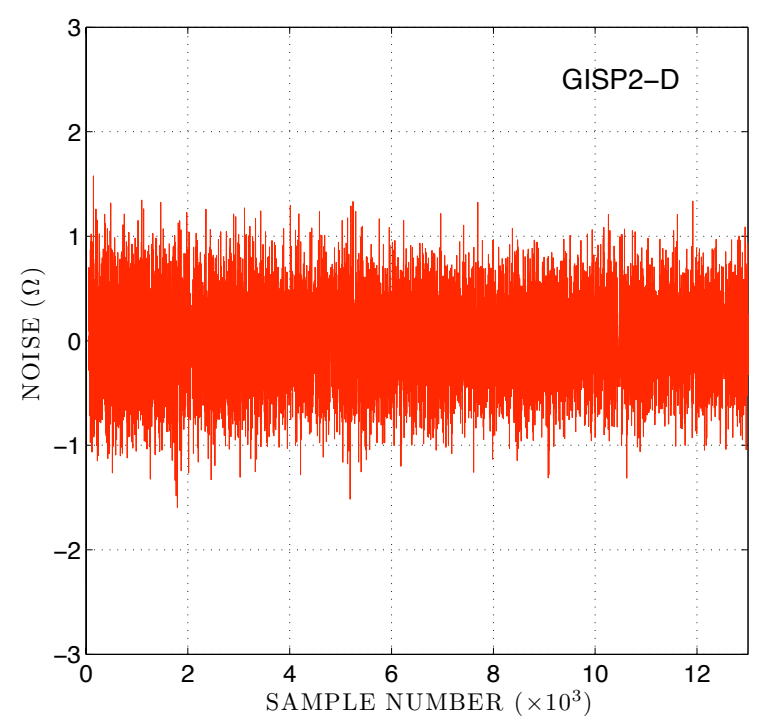

Figure 4. Raw noise for a typical logging experiment. This record was acquired from the nonconvecting portion of the GISP2-D borehole (central Greenland) without the use of hardware noise filters. In this example, the standard deviation of the noise was $\sigma_{R_{n}}=0.40 \Omega$ with sensor resistances $R_{s}$ ranging from 11.9 to $12.7 \mathrm{k} \Omega$.

thermistor packets are completely enclosed in a 4.0mm-diameter stainless-steel shell, allowing the probes to withstand the pressures encountered at $7-8 \mathrm{~km}$ in liquid-filled boreholes and the effects of corrosive chemicals such as n-butyl acetate (many of the deep boreholes drilled by the United States polar programs are filled with n-butyl acetate). The use of many small thermistor beads, glass encapsulation, and a high-conductivity steel shell all help to produce a high power-dissipation constant $P_{d}$. The $P_{d}$-value for these custom probes is $55 \mathrm{~mW} \cdot \mathrm{K}^{-1}$ in circulating xylene and is believed to be similar when logging through nbutyl acetate while the $\alpha_{T}$ values range from about $-0.045 \mathrm{~K}^{-1}$ at $0^{\circ} \mathrm{C}$ to $-0.065 \mathrm{~K}^{-1}$ at $-60^{\circ} \mathrm{C}$ (fig. 6 ). An inevitable disadvantage of this probe design is the relatively slow response time. In n-butyl acetate, the measured time constant is about 7 seconds. Five series of custom probes, each with a different $0^{\circ} \mathrm{C}$ resistance, are currently available to optimize the characteristics of the PTLS for any given experiment.

To convert sensor resistance to temperature, we use the 4-term calibration function

$$
T^{-1}=a_{0}+a_{1}\left(\ln R_{s}\right)+a_{2}\left(\ln R_{s}\right)^{2}+a_{3}\left(\ln R_{s}\right)^{3}
$$

where the constants $a_{i}$ are determined for each sensor 


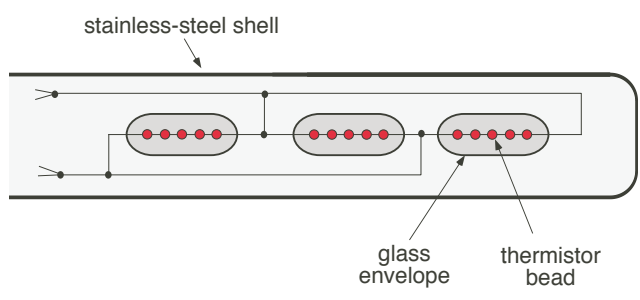

Figure 5. PTLS temperature sensor. The sensor consists of a parallel-series network of 15 small bead thermistors divided into three sealed packets. Beads extend over a 10-centimeter length within the 4-millimeter-diameter stainless-steel shell. The resistance readout measures the combined resistance of the parallel-series network.

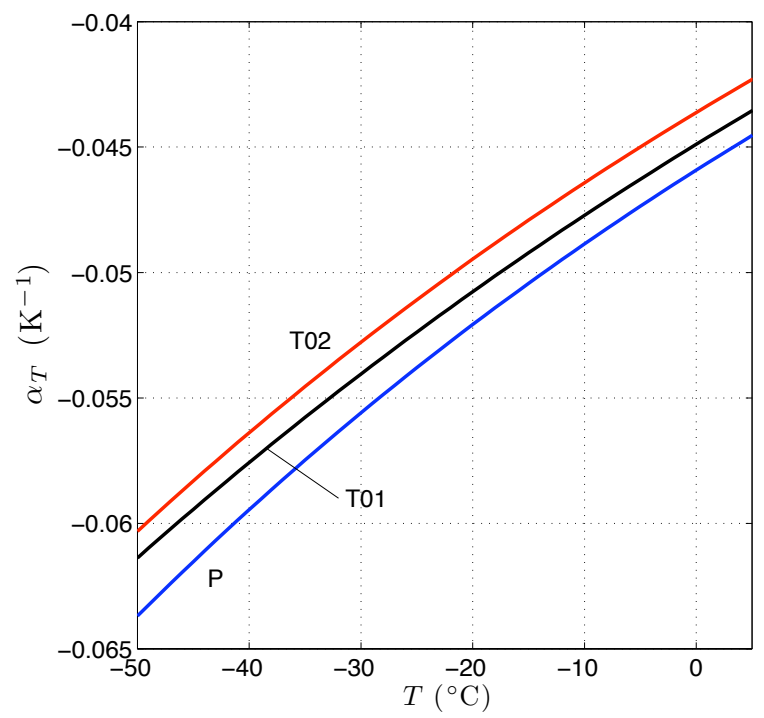

Figure 6. Temperature coefficient of resistance $\left(\alpha_{T}\right)$ for the P, T01, and T02 probe series used with the Polar Temperature Logging System. These probe series have a nominal resistance of $27 \mathrm{k} \Omega, 3.7 \mathrm{k} \Omega$, and $2.5 \mathrm{k} \Omega$ at $0^{\circ} \mathrm{C}$, respectively.

just before every field season in our thermal calibration facility, and $T$ is expressed in Kelvin. Equation (11) is an extension of the often-used 3-term Steinhart-Hart equation (Steinhart and Hart, 1968), which proves inadequate for our purposes. An F-test (for example, Bevington, 1969) demonstrates that a much better fit to our calibration data can be obtained with the 4-term function than with the standard Steinhart-Hart equation, particularly at temperatures below $0^{\circ} \mathrm{C}$. Sample temperature-calibration data and the resulting 4-term calibration fit (eq. 11) are shown in figure 7 .
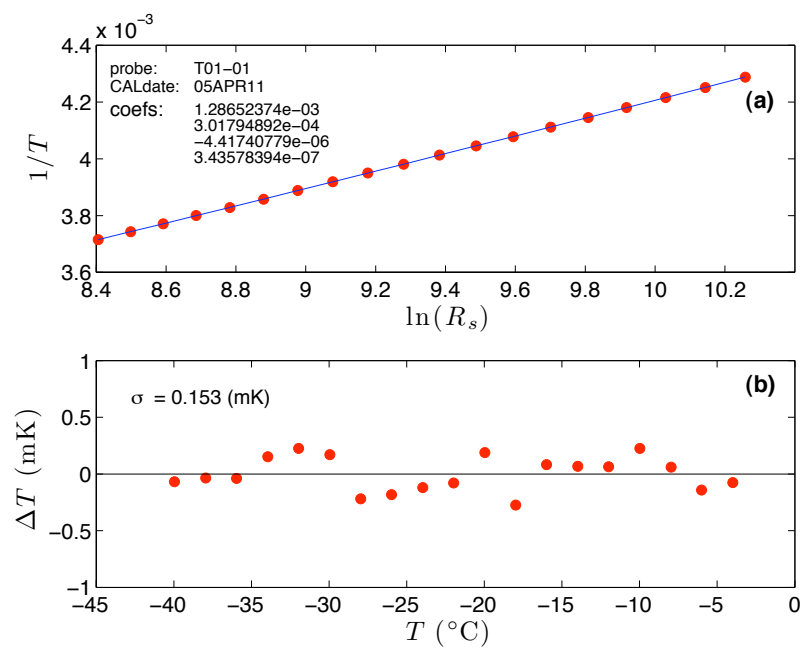

Figure 7. Sample calibration data for one of the temperature sensors (T01-01) along with the best 4-term SteinhartHart fit to the data (a). In this case, residuals from the fit to the calibration data have a standard deviation of 0.153 millikelvin (b).

The temperature resolution of the Kelvin circuit,

$$
\Delta T_{r} \approx \frac{\Delta R_{r}}{\alpha_{T} R_{s}}
$$

depends on the ratio of the smallest resistance resolvable by the resistance readout $\Delta R_{r}$ to the probe resistance $R_{s}$. Thus, to achieve sub-mK sensitivity, $\Delta R_{r} / R_{s}$ must be less than $5 \times 10^{-5}$. For the resistance readout currently used with the PTLS, the $\Delta R_{r} / R_{s}$ ratio ranges from $1.0 \times 10^{-5}$ (worst case) to $1.0 \times 10^{-6}$ (best case). The resulting temperature resolution is less than $0.2 \mathrm{mK}$ under all conditions (fig. 8). With the resistance readout used prior to $2008, \Delta T_{r}$ ranged from 0.1 to $1.1 \mathrm{mK}$; by judicious selection of the temperature probe for pre-2008 logging experiments, the resolution could almost always be reduced to less than $0.6 \mathrm{mK}$.

\subsection{Depth-Measurement System}

\subsubsection{System Description}

Depth information is obtained by measuring the angular rotation $d \theta$ of a precision measuring wheel located within the winch assembly (fig. 1) that is pressed tightly against the logging cable. Wheel rotation is detected by an optical shaft encoder that transmits quadrature waveforms to a bidirectional counter. The counter then converts angular rotation to distance (or depth) using $d \tilde{z}=\left(R_{w}+r\right) d \theta$, where $r$ and $R_{w}$ are the 


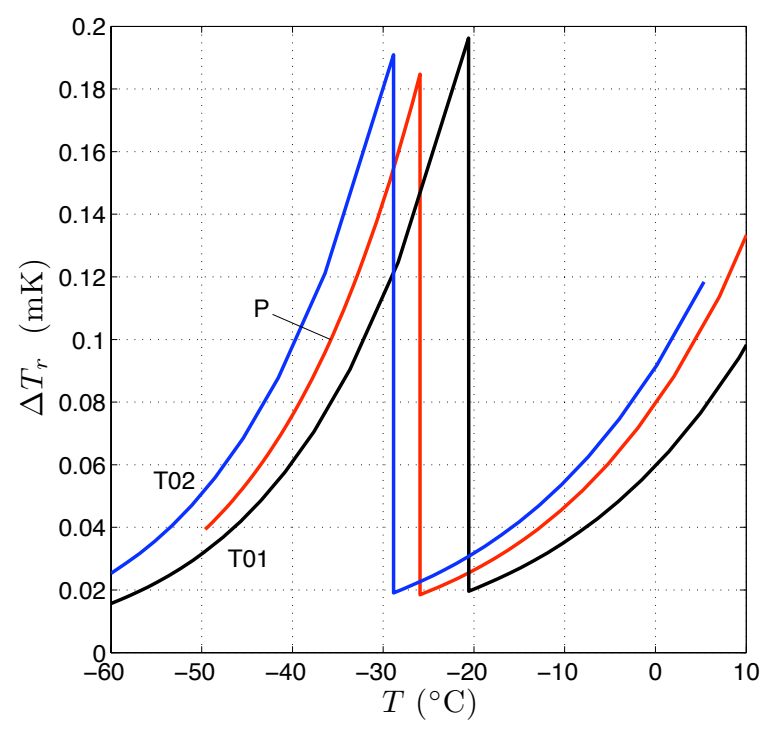

Figure 8. Temperature resolution of the current PTLS when using the P, T01, and T02 probe series.

radii of the logging cable and measuring wheel, respectively. The fundamental limit of the depth resolution is determined by the effective radius of the measuring system $\left(R_{w}+r\right)$ and the number of quadrature pulses output per revolution by the optical encoder. For the $4.5-\mathrm{km}$ system, this limit is $0.254 \mathrm{~cm}$. An additional limitation is imposed by the depth counter. For the 6 digit counter currently used with the PTLS, the depth resolution $\Delta z_{r}$ is $1.0 \mathrm{~cm}$ over the system's full depth range, although it can be set to $\Delta z_{r}=0.30 \mathrm{~cm}$ when collecting depth-calibration data. Before 2007, $\Delta z_{r}$ was $0.3 \mathrm{~cm}$ for boreholes less than $545 \mathrm{~m}$ deep and $3.0 \mathrm{~cm}$ for deeper holes due to the limited buffer size of the bidirectional counter used at that time.

\subsubsection{Depth Corrections}

To account for the force-dependent strains affecting the depth-measuring system, a depth-calibration function $C_{d}$ is established for each unique logging environment. This is done by moving the logging cable downhole approximately $40 \mathrm{~m}$ and comparing the distance $\tilde{\mathcal{L}}$ reported by the depth counter with the distance $\mathcal{L}$ measured by a fiberglass surveying tape that has a low temperature coefficient of thermal expansion $\left(9.3 \times 10^{-6} \mathrm{~K}^{-1}\right) ; \mathcal{L}$ is taken to be a measure of the "true" distance. $C_{d}$ is then defined by

$$
C_{d}(\tilde{Z}) \equiv \frac{\mathcal{L}(\tilde{Z})}{\tilde{\mathcal{L}}(\tilde{Z})}-1
$$

where $\tilde{Z}$ is the depth of the sensor-end of the cable (according to the counter) when the $(\mathcal{L}, \tilde{\mathcal{L}})$-data are collected. Depth-calibration data are acquired with the sensor-end of the cable at multiple depths $\tilde{Z}_{i}$ spanning as much of a borehole's depth range as possible. Temperature changes in the measuring wheel and in the test section of logging cable also affect the calibration data. Although we attempt to maintain a consistent temperature during the calibration period, temperature changes do sometimes occur. To remove the temperature effects, we define thermally corrected $C_{d}$ values by

$$
\begin{aligned}
C_{d}\left(T_{w}^{\star}, \tilde{Z}_{i}\right) & =C_{d}\left(T_{w_{i}}, \tilde{Z}_{i}\right)-\left(\frac{R_{w}}{R_{n}}\right) e_{w r T_{w}} \\
& -\left(\frac{r}{R_{n}}\right) e_{c r T_{w}}-\alpha f_{T}\left(T_{w}^{\star}-T_{w_{i}}\right)
\end{aligned}
$$

where $T_{w}^{\star}$ is a reference calibration temperature and $T_{w_{i}}$ is the temperature at the measuring wheel when the sensor-end of the cable is at $\tilde{Z}_{i}$. The second and third terms on the right-handside of equation (14) account for changes in the effective radius of the measuring system due to thermal strains while the fourth term accounts for the thermally induced longitudinal strain in the cable as it enters the relatively cold, air-filled portion of the borehole. Assuming the temperature of the cable is approximately $T_{w_{i}}$ when passing over the measuring wheel, the radial strains in the wheel and cable $\left(e_{w r T_{w}}, e_{c r T_{w}}\right)$ are both given by

$$
e_{\mathrm{x} r T_{w}}=\sqrt{\frac{1+\beta\left(T_{w_{i}}-T_{w}^{\star}\right)}{1+\alpha\left(T_{w_{i}}-T_{w}^{\star}\right)}}-1
$$

where $\alpha$ and $\beta$ are the linear and volumetric coefficients of thermal expansion. Our measuring wheels are isotropic disks so that $\beta=3 \alpha$. Factor $R_{n}=m / \Delta \theta_{r}$ is the nominal radius used by the depth counter where $m$ is the counter's internal multiplier and $\Delta \theta_{r}$ is the encoder's angular resolution. Parameter $f_{T}$ is the fraction of the temperature difference $T_{w_{i}}-T(h)$ experienced by the cable during a calibration test where $T(h)$ is the temperature at the bottom of the air-filled portion of the borehole; $f_{T}$ is typically about 0.3 .

Once the thermally corrected $C_{d}$ values have been determined, a least-squares fit is made to

$$
\mathcal{F}\left(\tilde{Z}_{i}\right)=C_{d}\left(T_{w}^{\star}, \tilde{Z}_{i}\right)-C_{d}\left(T_{w}^{\star}, \tilde{Z}^{\star}\right)
$$


where $\tilde{Z}^{\star}$ is a reference calibration depth selected from one of the $\tilde{Z}_{i}$ values near the middle of the depth range; reference temperature $T_{w}^{\star}$ is generally taken to be the $T_{w_{i}}$ value for the calibration data acquired at $\tilde{Z}_{i}=\tilde{Z}^{\star}$. The depth-calibration function is then given by the sum of the reference $C_{d}$-value and the experimentally determined function $\mathcal{F}(\tilde{Z})$, which isolates the force-dependent effects of the depth-measuring system relative to $C_{d}\left(T_{w}^{\star}, \tilde{Z}^{\star}\right)$,

$$
C_{d}\left(T_{w}^{\star}, \tilde{Z}\right)=C_{d}\left(T_{w}^{\star}, \tilde{Z}^{\star}\right)+\mathcal{F}(\tilde{Z}) .
$$

Figure 9 shows an example $\mathcal{F}(\tilde{Z})$ determined from depth-calibration data acquired in the 1-km-deep Siple Dome A borehole in West Antarctica.

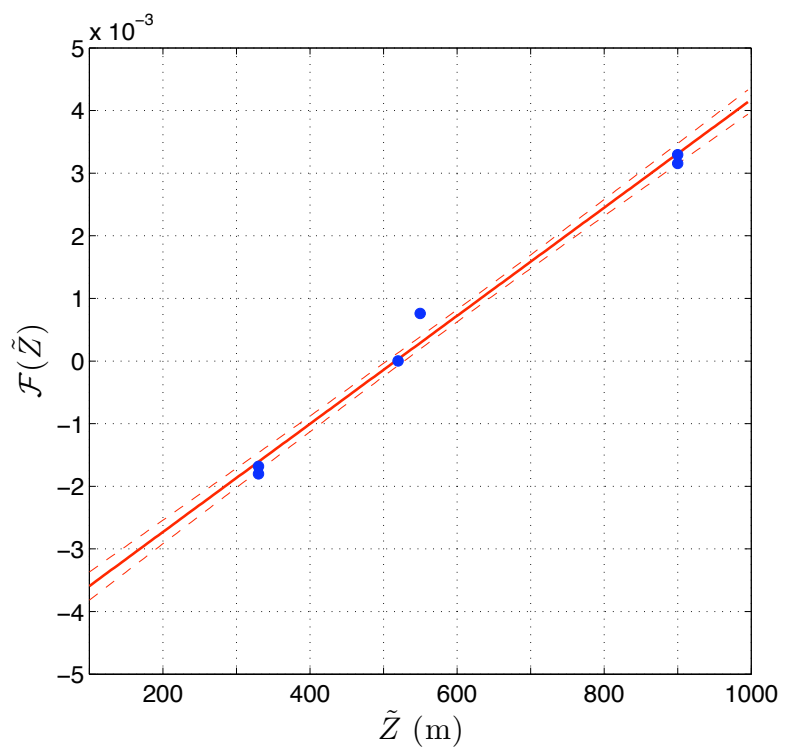

Figure 9. Least-squares fit to $\mathcal{F}\left(\tilde{Z}_{i}\right)$ values determined from depth-calibration data acquired in the Siple Dome A borehole, West Antarctica. Dashed lines indicate the uncertainty $( \pm 1 \sigma)$ of the least-squares fit.

With the availability of the $\mathcal{F}(\tilde{Z})$ and $C_{d}\left(T_{w}^{\star}, \tilde{Z}\right)$ functions, the total length of cable spooled into a borehole during a logging experiment can be found by integrating the distances $d \tilde{z}$ reported by the depth counter weighted by the depth-calibration function,

$$
Z \approx \int_{o}^{\tilde{Z}}\left[1+C_{d}\left(T_{w}^{\star}, \tilde{Z}-\tilde{z}\right)\right] d \tilde{z} .
$$

Substituting from equation (17) and letting

$$
\delta Z_{F}=\int_{0}^{\tilde{Z}} \mathcal{F}(\tilde{Z}-\tilde{z}) d \tilde{z}
$$

be the correction for the force-dependent effects, the length $Z$ becomes

$$
Z \approx\left[1+C_{d}\left(T_{w}^{\star}, \tilde{Z}^{\star}\right)\right] \tilde{Z}+\delta Z_{F} .
$$

This estimate accounts for most of the tension-induced radial and longitudinal strains within the logging cable and mechanical strains in the logging winch. However, a number of additional corrections must be made to obtain an estimate of the true sensor depth during a logging experiment. These include a correction for the buoyancy of the logging tool when immersed in the borehole fluid, for temperature changes near the measuring wheel affecting the radius of the depthmeasuring system, and for thermal strain in the cable as it moves downhole.

The correction for tool buoyancy consists of a simple offset that occurs when the logging tool enters the borehole fluid:

$$
\delta Z_{c l F}^{b t}= \begin{cases}0 & Z<h \\ K g\left(m_{t}^{f}-m_{t}^{a}\right)(h+\Delta x), & Z>h\end{cases}
$$

$K$ is the elastic stretch coefficient of the cable, $g$ is the gravitational acceleration, $m_{t}^{f}$ and $m_{t}^{a}$ are the weights of the logging tool in the borehole fluid and in air, $h$ is the depth to the air/fluid interface in the borehole, and $\Delta x$ is the horizontal distance between the measuring wheel and the hole. When logging with any of our temperature sensors, $\delta Z_{c l F}^{b t}$ is less than the resolution of the depth system and can be ignored. However, other tools for which $\delta Z_{c l F}^{b t}$ may be larger (for example, sonic and optical loggers) are occasionally used with our logging winch, so we include the buoyancy correction for completeness.

The temperature of the measuring wheel during a logging experiment can be significantly different from the reference calibration temperature $T_{w}^{\star}$. If the temperature of the wheel is $T_{w}$, the radial strain $e_{w r T_{w}}$ in the wheel relative to its calibration state can be found from equation (15). Since $T_{w}$ may vary considerably during a long logging run, the correction for the thermally induced radial change of the wheel is found through an integration:

$$
\delta Z_{w r T}=\left(\frac{R_{w}}{R_{n}}\right) \int_{o}^{\tilde{Z}} e_{w r T_{w}}(\tilde{z}) d \tilde{z} .
$$

The corresponding thermally induced radial strain in the cable near the measuring wheel has a negligible effect $(<10$ parts per million [ppm]) on the estimated depths and can be ignored. 
In polar environments, the fluid and overlying air column in a borehole are typically much colder than conditions on the surface near the logging winch. Thus, the logging cable will contract as it moves beyond the measuring wheel and enters the borehole. By the time a section of cable reaches depth $z$, it will experience a thermally induced longitudinal strain,

$$
e_{c l T}(z)=\alpha\left[T(z)-T_{w}\right],
$$

relative to its length when it passed over the measuring wheel. Some of this strain is built into the depthcalibration function $C_{d}$. Subtracting this portion, the correction for the thermally induced longitudinal strain in the cable is

$$
\delta Z_{c l T}=\int_{h}^{Z} e_{c l T}(z) d z-\alpha f_{T}\left[T(h)-T_{w}^{\star}\right] Z .
$$

Applying these corrections to equation (20), our estimate of the true sensor depth during a logging experiment is

$$
\begin{aligned}
Z=[1 & \left.+C_{d}\left(T_{w}^{\star}, \tilde{Z}^{\star}\right)\right] \tilde{Z}+\delta Z_{F}+\delta Z_{c l F}^{b t} \\
& +\delta Z_{w r T}+\delta Z_{c l T} .
\end{aligned}
$$

For most situations, $\delta Z_{F}$ is the largest correction and $\delta Z_{c l T}$ is the second largest.

\section{Measurement Uncertainties}

A variety of factors influence the uncertainty of the quantities being measured by the PTLS. For the temperature-measurement process, the primary uncertainties include those related to the PTLS resistance readout, uncertainties in the corrections made for systematic errors in the Kelvin circuit measurements (eqs. 1, 2, 3, 8), and uncertainties in the temperature sensor calibration used to convert measured resistance to temperature. The primary uncertainties for the depth-measurement process are related to the depth corrections (eqs. 19, 21, 22, 24).

This section provides an analysis of the measurement uncertainties following CIPM guidelines (ISO, 1993b). It is assumed the resistance-measuring circuitry is located on the surface since the PTLS is normally operated in this mode; several of the uncertainties to be described are not present when using downhole measurement circuitry, although the temperaturerelated drift of the circuit potentially can be quite large. With the CIPM approach, the components of a measurand's uncertainty are classified according to the method used to evaluate them. Type A uncertainty evaluations are based on statistical analysis of a series of observations while Type B evaluations are performed "by other means" using sound scientific judgment. The information used in a Type B analysis may, for example, include a general knowledge or experience with an instrument or the behavior of a material, manufacturer's specifications, or calibration reports. The degree of uncertainty for each component is given in terms of the standard uncertainty $u_{i}$ that describes the interval within which a quantity should occur with 67 percent probability. The combined standard uncertainty of a measurement is obtained by combining the individual Type A and Type B standard uncertainties using the propagation of uncertainty law (Taylor and Kuyatt, 1994, Appendix A). We implement the propagation law using the "root-sum-of-squares" (RSS) method. Although some of the uncertainties to be discussed are quite small with the current version of the PTLS, they were in some cases substantially larger with previous PTLS versions and therefore are discussed for completeness.

\subsection{ITS-90 Temperature Uncertainties}

\subsubsection{Resistance Readout Uncertainties}

The PTLS generally utilizes a commercial readout to measure the resistance of a temperature sensor during a logging experiment. Sources of uncertainty related to the resistance readout include the uncertainty of the resistance standards used to calibrate the readout, nonlinearity across the resistance scales, and internal noise. Current field procedures require temperature logs be completed within 24 hours of the readout's latest calibration.

Resistance Standards. Immediately before each logging experiment, the resistance readout is calibrated using $10 \mathrm{k} \Omega$ and $100 \mathrm{k} \Omega$ DC resistance standards (Fluke $742 \mathrm{~A}$ ), and a $0 \Omega$ short. In preparation for making measurements at the fixed calibration points, the readout and resistance standards are warmed up and then maintained at $23 \pm 0.5^{\circ} \mathrm{C}$ for at least one hour. Once acquired, the calibration data are used to determine the coefficients in a quadratic function $f_{c}(R)$ which is used to correct the readout's measurements at other resistances. The uncertainties at the calibration points are found by combining the calibration uncertainty of the standards themselves $(0.5 \mathrm{ppm}$ for the $10-\mathrm{k} \Omega$ standard, $1.25 \mathrm{ppm}$ for the $100-\mathrm{k} \Omega$ standard) with the uncertainty associated with their long- 
term drift $\left(2.0 \mathrm{ppm} \cdot \mathrm{yr}^{-1}\right.$ for the $10-\mathrm{k} \Omega$ standard and $3.0 \mathrm{ppm} \cdot \mathrm{yr}^{-1}$ for the $100-\mathrm{k} \Omega$ standard). With annual recalibration, the combined standard uncertainty of the resistance standards (in normalized form) is $u_{s, n} \equiv$ $\left(u_{s} / R_{s}\right)=2.1 \mathrm{ppm}$ at $10 \mathrm{k} \Omega$ and $3.2 \mathrm{ppm}$ at $100 \mathrm{k} \Omega$. These uncertainties are propagated to other resistances using a function of the same form as $f_{c}(R)$.

A different set of resistance standards was used prior to 2008. These standards were periodically calibrated using an instrument with a standard uncertainty of $58 \mathrm{ppm}$. Long-term drift between calibrations introduced an additional 58 ppm of uncertainty, yielding a combined standard uncertainty for these older standards of $u_{s, n}=82 \mathrm{ppm}$ at both $10 \mathrm{k} \Omega$ and $100 \mathrm{k} \Omega$.

Resistance Readout's Short-Term Uncertainty. The quoted short-term accuracy of the resistance readout currently used with the PTLS is $\pm 0.25 \Omega$ for $R_{s}<5 \mathrm{k} \Omega$ and \pm 50 ppm for $5 \mathrm{k} \Omega \leq R_{s} \leq 200 \mathrm{k} \Omega$. This specification includes the nonlinearity of the readout across the measurement range and internal noise. According to the manufacturer, the accuracy $( \pm a)$ specifies the width of a rectangular (uniform) probability distribution function; this PDF is taken to have a corresponding standard uncertainty of $a / \sqrt{3}$ (Taylor and Kuyatt, 1994). Thus the short-term standard uncertainty of the readout is $u_{r}=0.14 \Omega$ for $R_{s}<5 \mathrm{k} \Omega$ and $u_{r, n} \equiv\left(u_{r} / R_{s}\right)=29 \mathrm{ppm}$ for $5 \mathrm{k} \Omega \leq R_{s} \leq 200 \mathrm{k} \Omega$. Before 2008, a different readout was used whose accuracy included a component for the full-scale resistance value $R_{f}$. For this instrument the short-term standard uncertainty was $u_{r, n}=11.6+5.8\left(R_{f} / R_{s}\right)$ ppm.

\section{Combined Resistance-Readout Uncertainty}

$\mathbf{u}_{\tilde{\mathbf{R}}_{\mathbf{s}}}$. Combining the uncertainties of the resistance standards with the short-term accuracy of the readout, we obtain the combined standard uncertainty $u_{\tilde{R}_{s}}$ of the resistance readout measurements. With the currently used resistance standards, $u_{\tilde{R}_{s}}$ is dominated by the short-term uncertainty of the readout itself. Thus $u_{\tilde{R}_{s}}=0.14 \Omega$ for $R_{s}<5 \mathrm{k} \Omega$. For the more common sensor resistances $\left(R_{s} \geq 5 \mathrm{k} \Omega\right)$, the combined standard uncertainty is $u_{\tilde{R}_{s}, n} \equiv\left(u_{\tilde{R}_{s}} / R_{s}\right)=29 \mathrm{ppm}$. Prior to 2008 the uncertainty of the standards was the major contributor to $u_{\tilde{R}_{s}}$ at most resistances, leading to combined readout uncertainties $u_{\tilde{R}_{s}, n}$ ranging from 83 to $107 \mathrm{ppm}$. Since both of the uncertainty components $\left(u_{s}, u_{r}\right)$ are classified as Type B, the combined uncertainty of the resistance readout is also Type B.

\subsubsection{Kelvin Circuit Uncertainties}

As discussed in Section 2.2, the Kelvin circuit monitored by the resistance readout introduces multiple sources of systematic error in the resistance measurements. These sources include leakage paths between the circuit's electrical conductors, capacitance effects, self-heating effects, and thermal EMFs. Although we correct for the systematic errors, there are uncertainties associated with the corrections. In addition, electrostatic coupling, electromagnetic EMFs, triboelectric currents, and switching effects within the slipring assembly all generate noise in the Kelvin circuit. This noise is largely removed during data processing by using 1D wavelet denoising techniques (Misiti and others, 2005). However, because the noise removal process is imperfect, there remains an uncertainty in the resistance measurements associated with the noise.

Leakage Paths. Applying the propagation of uncertainty law to the leakage path correction $\delta R_{l}$ (eq. 1 ), the standard uncertainty $u_{l}$ of the leakage correction is given by

$$
u_{l}=\frac{R_{s}}{\left(R_{s}+R_{l}\right)^{2}} \sqrt{\left(R_{s}+2 R_{l}\right)^{2} u_{R_{s}}^{2}+R_{s}^{2} u_{R_{l}}^{2}}
$$

where $u_{R_{l}}$ is the standard uncertainty of leakage resistance $R_{l}$. Since $R_{l} \gg R_{s}$ for all operational conditions, we can re-express the leakage-path uncertainty in the normalized form

$$
u_{l, n} \equiv\left(\frac{u_{l}}{R_{s}}\right)=\sqrt{4\left(\frac{u_{R_{s}}}{R_{l}}\right)^{2}+\left(\frac{R_{s}}{R_{l}}\right)^{2}\left(\frac{u_{R_{l}}}{R_{l}}\right)^{2}} .
$$

The leakage resistance $R_{l}$ is always $\geq 10 \mathrm{G} \Omega$ for the PTLS while the standard uncertainty of its determination is $u_{R_{l}} \approx 11.5 \mathrm{G} \Omega$ based on the specifications of the measuring instrument. With such high $R_{l}$ values, the second term within the square root of equation (27) exceeds the first term by about $10^{8}$. Thus the standard uncertainty of the leakage correction reduces to

$$
u_{l, n}=\left(\frac{R_{s}}{R_{l}}\right)\left(\frac{u_{R_{l}}}{R_{l}}\right) .
$$

Capacitance Effects. The standard uncertainty $u_{c}$ of the capacitance correction $\delta R_{c}$ (eq. 2) is given by

$$
u_{c}=\tau R^{\prime} \sqrt{\left(\frac{u_{R_{s}}}{R_{s}}\right)^{2}+\left(\frac{u_{C}}{C}\right)^{2}+\left(\frac{u_{R^{\prime}}}{R^{\prime}}\right)^{2}}
$$

where $R^{\prime}=\partial R_{s} / \partial t$ is the rate of resistance change while logging downhole. The accuracy of the instrument used to measure the capacitance of the logging 
cable, or of any filtering capacitors, establishes the relative capacitance uncertainty $\left(u_{C} / C\right)$. Based on the manufacturer's specifications and a rectangular PDF, $\left(u_{C} / C\right) \simeq 0.0074$. This term completely dominates $\left(u_{R_{s}} / R_{s}\right)$, which is of the order $10^{-4}$ or smaller. Using a Taylor Series expansion, the relative uncertainty of $R^{\prime}$ is found to be

$$
\left(\frac{u_{R^{\prime}}}{R^{\prime}}\right) \approx \frac{1}{\sqrt{3}}\left(\frac{1}{m}+\frac{\Delta t_{r}}{\Delta t}\right)
$$

where $\Delta t$ is the sampling rate, $\Delta t_{r}$ is the resolution of the time measurements (determined by the computer's clock), and $m$ is a measure of the resistance change between samples relative to the resolution $\Delta R_{r}$ of the resistance readout,

$$
m=\frac{R_{i+1}-R_{i-1}}{2 \Delta R_{r}} .
$$

Our sampling rates are slow enough that $\Delta t_{r} / \Delta t \ll$ $1 / m$ under all circumstances. Dropping negligible terms, the standard uncertainty of the capacitance correction becomes

$$
u_{c, n} \equiv\left(\frac{u_{c}}{R_{s}}\right)=\frac{C \Delta R_{r}}{\Delta t} \sqrt{\frac{1}{3}+m^{2}\left(\frac{u_{C}}{C}\right)^{2}} .
$$

Self-Heating Effects. For the self-heating correction $\delta R_{h}$ (eq. 3), the associated standard uncertainty $u_{h}$ is given by

$u_{h}=\frac{\alpha_{T} I_{s}^{2} R_{s}^{2}}{P_{d}} \sqrt{4\left(\frac{u_{I_{s}}}{I_{s}}\right)^{2}+\left(\frac{u_{R_{s}}}{R_{s}}\right)^{2}+\left(\frac{u_{P_{d}}}{P_{d}}\right)^{2}}$.

The relative uncertainty of the power dissipation constant $\left(u_{P_{d}} / P_{d}\right)$ is estimated to be roughly 0.1 , which is several orders of magnitude larger than either $\left(u_{I_{s}} / I_{s}\right)$ or $\left(u_{R_{s}} / R_{s}\right)$. Dropping negligible terms, the uncertainty of the self-heating correction is

$$
u_{h, n} \equiv\left(\frac{u_{h}}{R_{s}}\right)=\frac{\alpha_{T} I_{s}^{2} R_{s}}{P_{d}}\left(\frac{u_{P_{d}}}{P_{d}}\right) .
$$

Thermal EMFs. The standard uncertainty $u_{e}$ of the thermal EMF correction $\delta R_{e}$ (eq. 8) is

$$
u_{e}=\frac{1}{I_{s}} \sqrt{u_{V_{\mathrm{emf}}}^{2}+V_{\mathrm{emf}}^{2}\left(\frac{u_{I_{s}}}{I_{s}}\right)^{2}} .
$$

Since the relative uncertainty of the regulated test current $I_{s}$ is several orders smaller than that of the thermoelectric voltages, the standard uncertainty of the thermal EMF correction is simply

$$
u_{e, n} \equiv\left(\frac{u_{e}}{R_{s}}\right)=\frac{u_{V_{e m f}}}{I_{s} R_{s}} .
$$

Prior to 2008, $u_{V_{\mathrm{emf}}}$ was estimated to be about the same magnitude as $V_{\text {emf }}(\sim 0.5 \mu \mathrm{V})$. However, with the current PTLS design, $V_{\mathrm{emf}}$ is essentially zero and thus, so is $u_{V_{\text {emf }}}$ and $u_{e}$.

Noise. Extensive tests with noisy synthetic data show that the wavelet denoising methods used during data processing reduce the noise in the recorded resistances by a factor of 8 . Thus the standard uncertainty of the resistance measurements due to instrumental noise is $u_{n} \approx \sigma_{\tilde{R}_{n}} / 8$ where $\sigma_{\tilde{R}_{n}} \leq \sigma_{R_{n}}$ (see discussion, Section 2.2.3).

Summary of Kelvin Circuit Uncertainties. Equations 28, 32, 34, and 36 indicate the uncertainties $\left(u_{l}, u_{c}, u_{h}, u_{e}\right)$ of the Kelvin-circuit resistance corrections depend on the specific conditions during a temperature $\log$ and thus must be treated on a case-bycase basis. Despite the need to specifically consider the conditions for each experiment when evaluating the uncertainties, some general statements can be made. (1) Of the resistance-correction uncertainties, the uncertainty of the leakage-path correction $\left(u_{l}\right)$ is the largest with the current PTLS operating under normal conditions (fig. 10). (2) Without the use of the resistance readout's internal filters or a filtering capacitor, the uncertainty $u_{n}$ due to noise is the dominant Kelvincircuit uncertainty for sensor resistances less than 20$30 \mathrm{k} \Omega$. Even with the use of hardware filters to reduce the noise uncertainty, $u_{n}$ is generally still the dominant uncertainty at low resistances $\left(R_{s}<20 \mathrm{k} \Omega\right.$ ). (3) At high resistances $\left(R_{s}>70 \mathrm{k} \Omega\right)$, the uncertainty of the leakage-path correction $\left(u_{l}\right)$ is the dominant uncertainty. (4) All the uncertainties associated with the resistance corrections and noise are less than $0.1 \mathrm{mK}$ for sensor resistances in the range $10-170 \mathrm{k} \Omega$. (5) The uncertainties $\left(u_{l}, u_{c}, u_{h}, u_{e}\right)$ associated with the Kelvin-circuit resistance corrections are all classified as Type B uncertainties while the noise uncertainty $u_{n}$ is Type A.

\subsubsection{Temperature-Sensor Calibration Uncer- tainties}

Before every set of field experiments, the PTLS temperature sensors are calibrated on the ITS-90 temperature scale (Mangum and Furukawa, 1990) at the USGS thermal calibration facility in Lakewood, Colorado. Temperatures on the ITS-90 scale are defined 


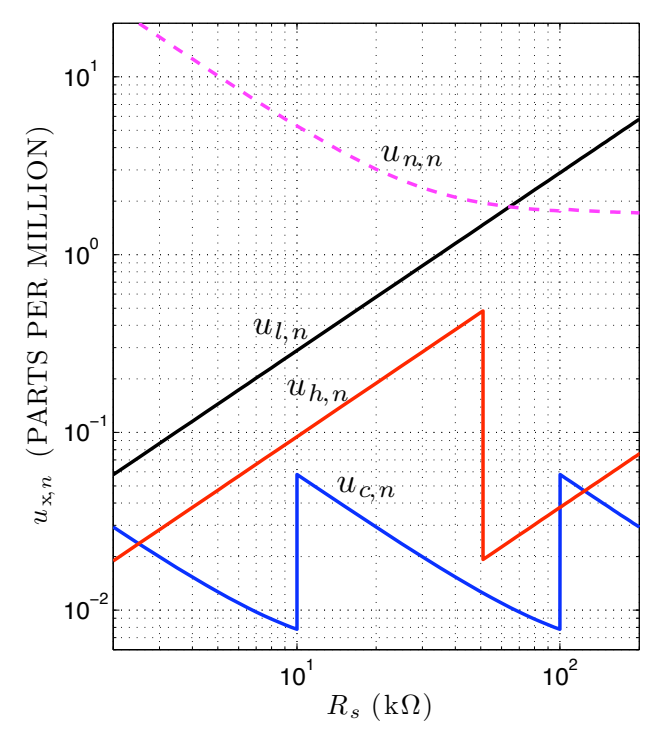

Figure 10. Standard uncertainties $\left(u_{l}, u_{c}, u_{h}\right)$ associated with the resistance corrections, displayed in normalized form $u_{\mathrm{x}, n} \equiv\left(u_{\mathrm{x}} / R_{s}\right)$, for typical operating conditions. In this example, the uncertainty of the capacitance correction $\left(u_{c}\right)$ corresponds to the case shown in figure 3 ( $v=5.5$ centimeters per second, $\partial T / \partial z=25$ millikelvins per meter, $\tau=10$ milliseconds, $\left.C=\tau / R_{s}\right)$. The uncertainty of the thermal EMF correction $\left(u_{e}\right)$ is essentially zero with the current PTLS design. The noise uncertainty $\left(u_{n}\right)$ shown by the dashed line represents an upper bound for most operating conditions; it assumes hardware noise filters are not used. Equivalent temperature uncertainties are given by $u_{\mathrm{x}, n} / \alpha_{T}$.

in terms of a set of fixed points (melting, boiling, and triple points of pure substances), interpolating instruments, and equations relating the property measured by each interpolating instrument to temperature. Between $13.8 \mathrm{~K}$ and $1,235 \mathrm{~K}$, the official interpolating instrument is the standard platinum resistance thermometer (SPRT). Thus, equations describing how the resistance of a standard SPRT varies with temperature are embodied in the definition of the ITS-90 temperature scale. To calibrate the PTLS temperature sensors, we use a 25.5- $\Omega$ quartz-sheath SPRT as our local standard. The probes to be calibrated are inserted into a copper equilibration block that is immersed in a temperature-controlled fluid bath; the SPRT is positioned in the equilibration block at the same distance from the center as the probes. The copper block effectively damps short-term temperature fluctuations in the calibration bath and improves the uniformity of the thermal field surrounding the probes and the SPRT. Once the bath stabilizes at a predetermined calibration point, the data-acquisition system simultaneously acquires the SPRT reference temperature $T^{\star}$ and the resistance $R^{\star}$ of each temperature sensor being tested. This process is repeated across the entire calibration range in $2-\mathrm{K}$ increments with upcoming field experiments determining the calibration limits. Total least squares is then used to find the constants $\left(a_{i}\right)$ in the 4-term calibration function (eq. 11) from the $\left(T^{\star}, R^{\star}\right)$ calibration data. Residuals from this fit typically have standard deviations ranging from 0.20 to $0.45 \mathrm{mK}$.

The uncertainty of the resulting PTLS temperature-sensor calibrations is determined by a number of factors, including: the uncertainty of the SPRT calibration at ITS-90 fixed points, the propagation of error between those points, the accuracy of the SPRT readout, the accuracy of the thermistor scanner, and the magnitude of the temporal and spatial temperature variations within the calibration bath. The residuals from the least-squares fit to the calibration data do not reflect many aspects of the total uncertainty of the temperature-sensor calibration.

SPRT Reference Temperatures $T^{\star}$. The uncertainty of the SPRT reference temperature $T^{\star}$ is determined by the uncertainty of the SPRT calibration and the accuracy of the instrument (readout) used to monitor the SPRT temperatures. Our quartz SPRT was most recently calibrated by Hart Scientific (American Fork, Utah) who provided expanded uncertainties (coverage factor $k=2$ ) at fixed calibration points between $-200^{\circ} \mathrm{C}$ and $0^{\circ} \mathrm{C}$. The equivalent standard uncertainties are $0.5 \mathrm{mK}$ at $-197^{\circ} \mathrm{C}, 0.2 \mathrm{mK}$ at $-38.8344^{\circ} \mathrm{C}$ (triple point of mercury), and $0.1 \mathrm{mK}$ at $0.010^{\circ} \mathrm{C}$ (triple point of water). Because the definition of the ITS-90 temperature scale over this range is based on equations describing the behavior of a standard SPRT, the calibration uncertainties between the fixed points are of the same order as those at the adjacent fixed points. We use error propagation curves provided by the National Institute of Standards and Technology to determine how the uncertainties at the triple point of mercury (TPHg) and triple point of water (TPW) propagate to other temperatures. Combining the propagated $\mathrm{TPHg}$ and TPW uncertainties, the standard uncertainty of the SPRT calibration ranges from $0.27 \mathrm{mK}$ at $-60^{\circ} \mathrm{C}$ to $0.10 \mathrm{mK}$ at $0.01^{\circ} \mathrm{C}$.

The accuracy of the SPRT readout is $\pm 0.0005 \Omega$ for SPRT resistances less than $25 \Omega$ and $\pm 20 \mathrm{ppm}$ of the 
reading at higher resistances. Based on the thermal response of SPRTs (McGee, 1988), these specifications translate to a standard uncertainty ranging from $2.77 \mathrm{mK}$ at $-60^{\circ} \mathrm{C}$ to $2.90 \mathrm{mK}$ at $0^{\circ} \mathrm{C}$. Combining the uncertainty of the SPRT calibration with that of the SPRT readout, we obtain the standard uncertainty $u_{T^{*}}$ of the SPRT reference temperatures. The combined uncertainty $u_{T^{\star}}$ is clearly dominated by the uncertainty of the SPRT readout with values ranging from $2.79 \mathrm{mK}$ at $-60^{\circ} \mathrm{C}$ to $2.90 \mathrm{mK}$ at $0^{\circ} \mathrm{C}$ (fig. 11). Since the uncertainty of the SPRT calibration and the SPRT readout are both evaluated using Type B methods, the uncertainty of the SPRT temperature measurements is also classified as Type B.

Temperature-Sensor Resistance Measurements $\boldsymbol{R}^{\star}$. The resistance of the PTLS temperature sensors being calibrated is monitored using an 8-channel thermistor scanner whose accuracy is $\pm 100 \mathrm{ppm}$ of the reading. Based on a uniform PDF, the corresponding standard uncertainty $u_{R^{\star}}$ of the scanner's resistance measurements is $58 \mathrm{ppm}$. Expressed in terms of temperature, $u_{R^{\star}}$ ranges from $0.88 \mathrm{mK}$ at $-60^{\circ} \mathrm{C}$ to $1.28 \mathrm{mK}$ at $0^{\circ} \mathrm{C}$ for the $\mathrm{T} 01$ probe series; $u_{R^{\star}}$ is approximately 3 percent less for the $\mathrm{P}$ probe series and 2 percent more for the T02 probes (fig. 11). This is a Type B uncertainty. As with the SPRT readout, the thermistor scanner is operated within the $18-28^{\circ} \mathrm{C}$ range in order to achieve full accuracy.

Calibration Bath and High-Conductivity Equilibration Block. Other sources that affect the uncertainty of the temperature-sensor calibrations include temporal and spatial variations of the thermal field within the calibration bath. Both of these sources can cause temperature offsets between the probes being calibrated and the reference SPRT. The stability of the calibration bath (Hart model 7060) is reported to be $\pm 2.5 \mathrm{mK}$ at $-60^{\circ} \mathrm{C}$. This figure represents an expanded uncertainty with coverage factor $k=2$. Experiments at $-20^{\circ} \mathrm{C}$ confirm this value. Thus we take the stability of the bath to be $\pm 2.5 \mathrm{mK}$ across the full calibration range; the corresponding standard uncertainty $u_{b_{f}}$ of the bath fluctuations is $1.25 \mathrm{mK}$. Several experiments were done to determine the nonuniformity of the thermal field in the central portion of the bath where the calibrations are performed. We find that the standard uncertainty $u_{b_{u}}$ of the spatial variations in this region is $2.5 \mathrm{mK}$.

To further control the stability and uniformity of the thermal field experienced by the probes and SPRT during calibration, we use a 7.62 -cm-diameter, high-

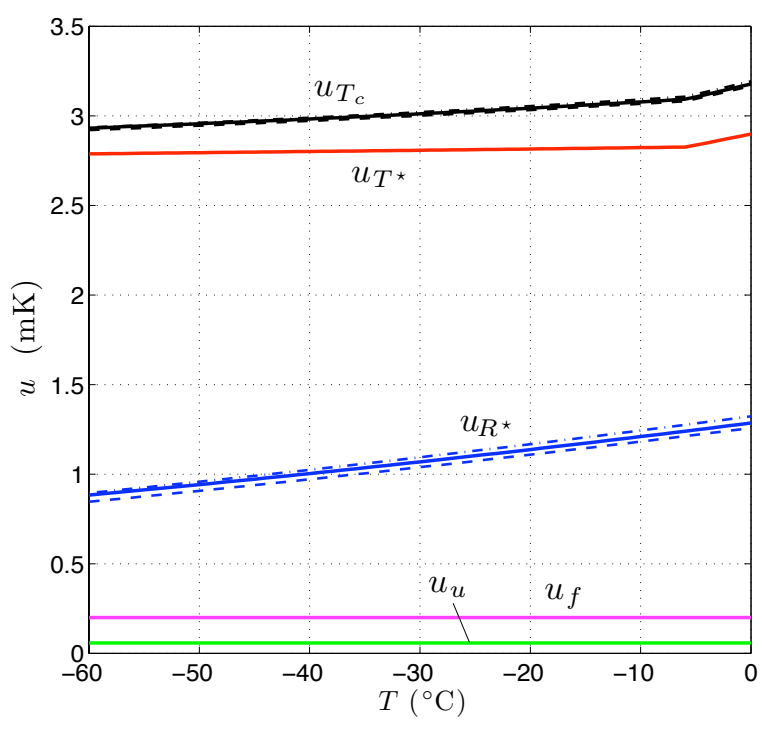

Figure 11. Combined standard uncertainty $u_{T_{c}}$ of the PTLS temperature-sensor calibration. Also shown are the standard uncertainties of the individual components: $u_{T^{*}}$ is the uncertainty of the SPRT reference temperature, $u_{R^{\star}}$ is the uncertainty of the temperature-sensor resistance measurements, $u_{f}$ is the uncertainty associated with temperature fluctuations in the calibration bath, and $u_{u}$ is the uncertainty due to bath nonuniformity. The three $u_{R^{\star}}$ curves (dashed, solid, dash-dot) show the uncertainty of the resistance measurements for the $\mathrm{P}, \mathrm{T} 01$, and $\mathrm{T} 02$ probe series, respectively.

conductivity (copper) equilibration block within the calibration bath. The SPRT and PTLS probes are inserted in tight-fitting holes located $2.90 \mathrm{~cm}$ from the center. Heat-transfer simulations show that thermal fluctuations in the bath with periods less than 0.1 minute are completely damped at the position of the probes, while those with periods of 3 minutes (where the largest bath fluctuations occur) are damped by a factor of about 0.4 (function $f$, fig. 12). These fluctuations would not be an issue if the PTLS probes and the SPRT had identical time constants so that they would synchronously warm and cool in response to the temperature fluctuations. However, the time constant of the SPRT (18.5 seconds) is much longer than that of the PTLS temperature sensors $(4.0 \mathrm{sec}-$ onds) in this situation. Convolving the response functions of the SPRT and the PTLS temperature sensors (Saltus and Clow, 1994) with synthetic temperature fluctuations shows that the maximum temperature discrepancy between the SPRT and the probes caused by 
dissimilar time constants occurs at a period of about 0.8 minute (function $g$, fig. 12). However, little power occurs at such short periods due to the damping of the block. Considering the joint effects of equilibration block damping and the dissimilar time constants, the largest discrepancies between the recorded SPRT temperatures and the probe temperatures occur at periods of 2-3.5 minutes (function $f \cdot g$, fig. 12 ). This coincidentally matches the period where the bath fluctuations have their greatest power. The resulting standard uncertainty in temperature-sensor calibration related to bath fluctuations is $u_{f}=(0.16) u_{b_{f}}=0.20 \mathrm{mK}$.

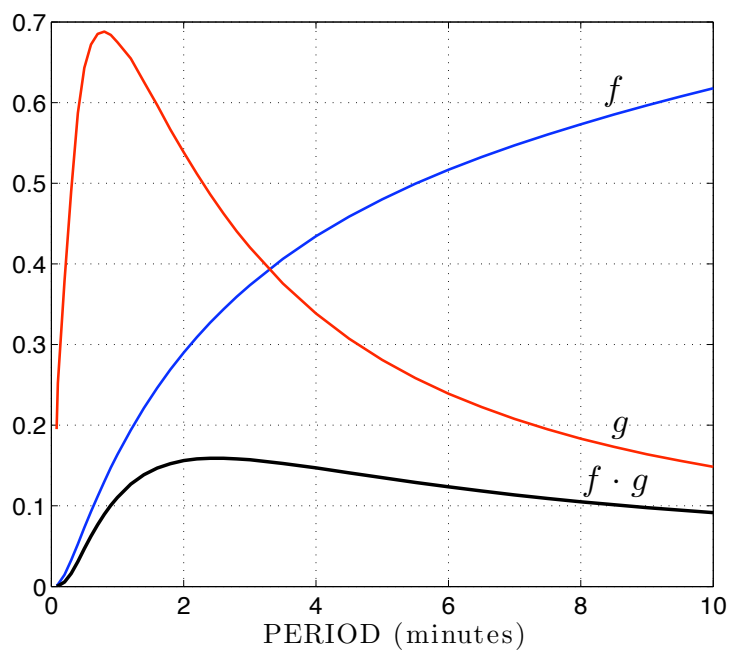

Figure 12. Damping of temperature fluctuations in the calibration bath by the high-conductivity equilibration block. Function $f=\left(u_{p} / u_{b_{f}}\right)$ describes the relative damping provided by the block; $u_{b_{f}}$ is the magnitude of the bath fluctuations while $u_{p}$ is the magnitude of the fluctuations inside the block near the probes and SPRT. The block is essentially opaque to short-period fluctuations ( $<0.1$ minute) but is transparent to periods longer than 30 minutes. Function $g=\left(u_{f} / u_{p}\right)$ shows the relative temperature discrepancy between the SPRT and the probes caused by their dissimilar time constants. Combining the block damping with the dissimilar time-constant effect, the standard uncertainty of the temperature-sensor calibration due to bath fluctuations is $u_{f}=(f \cdot g) u_{b_{f}}$. The product $(f \cdot g)$ reaches a maximum value of 0.16 at periods of $2-$ 3.5 minutes.

Persistent temperature gradients always exist to some extent in calibration baths due to imperfect mixing of the bath fluid. Experiments with the Hart 7060 bath show that the standard uncertainty of the thermal field in the vicinity of the equilibration block is $u_{b_{u}}=2.5 \mathrm{mK}$. However, the field inside the block is expected to be much more uniform. To quantify the uniformity, the resistance change of an array of thermistors located within the block was monitored while the block was rotated about its central axis; the bath was held at a fixed temperature (to within $u_{b_{f}}$ ) during these tests. After removing temporal fluctuations due to bath instability, the thermal field was found to be uniform to within $\pm 0.10 \mathrm{mK}$ inside the block at the location of the probes. The primary uncertainty of this determination is associated with the noise of the thermistor scanner (roughly $\pm 0.04 \mathrm{mK}$ ) as the experiment effectively removed uncertainties associated with the calibration of the individual thermistors; calibration, long-term drift, and nonlinearity of the thermistor scanner; and bath stability. The standard uncertainty of the temperature-sensor calibration related to bath nonuniformity is then estimated to be $u_{u}=0.10 / \sqrt{3}=$ $0.06 \mathrm{mK}$. Both bath uncertainties $\left(u_{f}, u_{u}\right)$ are classified Type A.

Combined Temperature-Sensor Calibration Uncertainty $\boldsymbol{u}_{\boldsymbol{T}_{\mathrm{c}}}$. Combining the uncertainty of the SPRT temperature measurements, of the thermistor scanner, and of the bath variations, the combined standard uncertainty $u_{T_{c}}$ of the temperature-sensor calibrations ranges from $2.93 \mathrm{mK}$ at $-60^{\circ} \mathrm{C}$ to $3.18 \mathrm{mK}$ $0^{\circ} \mathrm{C}$ (fig. 11). Although the thermistor scanner and the bath variations contribute to $u_{T_{c}}$, the uncertainty of the SPRT temperature is the dominant factor in the combined calibration uncertainty. A small dependence of $u_{R^{\star}}$ on the $\alpha_{T^{-}}$-characteristics of each probe series produces about a 1 percent difference in the combined uncertainty at any given temperature; this effect is small enough that it can be ignored. Because both Type A and Type B methods were used to evaluate the component uncertainties, the combined calibration uncertainty $u_{T_{c}}$ is classified as Type A,B.

\subsubsection{Combined ITS-90 Temperature Uncertainties}

Combining the resistance-readout uncertainty $\left(u_{\tilde{R}_{s}}\right)$, the resistance-correction uncertainties $\left(u_{l}, u_{c}\right.$, $\left.u_{h}, u_{e}\right)$, the uncertainty due to instrumental noise $\left(u_{n}\right)$, and the temperature-sensor calibration uncertainty $\left(u_{T_{c}}\right)$, we finally obtain the total standard uncertainty $u_{T}$ of the PTLS temperature-measurement process. In order to express $u_{T}$ in terms of temperature, most of the component uncertainties $\left(u_{R}, u_{l}, u_{c}, u_{h}, u_{e}, u_{n}\right)$ must be converted from resistances, where they are more naturally defined, to temperatures. This conversion involves the $\alpha_{T}$-characteristics of the temper- 
ature sensors. Thus, the total uncertainty of the temperature-measurement process depends to some extent on which probe is used during a logging experiment. This is particularly true at warm temperatures due to the degradation of the resistance readout's accuracy at resistances less than $5 \mathrm{k} \Omega$ (fig. 13). To avoid this degradation, we strive always to select a probe whose resistance will remain above $5 \mathrm{k} \Omega$ for the duration of an experiment; thus the T02 probes, for example, are used only at temperatures below $-15^{\circ} \mathrm{C}$ while the $\mathrm{P}$-series probes can be used up to at least $0^{\circ} \mathrm{C}$. With this constraint, the standard uncertainty of the temperature-measurement process ranges from $3.0 \mathrm{mK}$ at $-60^{\circ} \mathrm{C}$ to $3.3 \mathrm{mK}$ at $0^{\circ} \mathrm{C}$ with the current PTLS. As shown in figure 13, the total temperature uncertainty $u_{T}$ is strongly dominated by the temperaturesensor calibration uncertainty $u_{T_{c}}$ with the current PTLS design. The resistance readout contributes $0.5-$ $0.6 \mathrm{mK}$ to the total while the upper limit of the noise contribution is generally $\leq 0.25 \mathrm{mK}$. Individual resistance-correction uncertainties $\left(u_{l}, u_{c}, u_{h}, u_{e}\right)$ are less than $0.1 \mathrm{mK}$ under all normal conditions. The combined uncertainty $u_{T}$ of the ITS-90 temperature measurements is a Type A,B uncertainty.

Several of the temperature uncertainty components were larger before 2008, especially the resistance readout uncertainty $u_{\tilde{R}_{s}}$, the uncertainty of the thermal EMF correction $\left(u_{e}\right)$, and the uncertainty of the capacitance correction $\left(u_{c}\right)$. During 1993-2007, $u_{\tilde{R}_{s}}$ was about 3 times larger $(1.4-2.1 \mathrm{mK})$ than with the current system. In addition, the thermal EMF uncertainty was comparable to the noise uncertainty $u_{n}$ rather than being zero. Although larger, $u_{c}$ was still less than $0.03 \mathrm{mK}$ and thus of little consequence. Combining all the components, the total standard uncertainty $u_{T}$ of the temperature-measurement process during 19932007 ranged from $3.2 \mathrm{mK}$ at $-60^{\circ} \mathrm{C}$ to $3.8 \mathrm{mK}$ at $0^{\circ} \mathrm{C}$.

\subsection{Relative Temperature Uncertainties}

Section 3.1 focused on the standard uncertainty $u_{T}$ of the PTLS temperature measurements relative to the ITS-90 absolute temperature scale. For climatechange detection, this is the appropriate measure of uncertainty. However when reconstructing past climatic changes using borehole paleothermometry, we are not as concerned about uncertainties relative to international absolute measurement scales as we are about the potential distortion of a temperature profile by measurement errors that could be misinterpreted

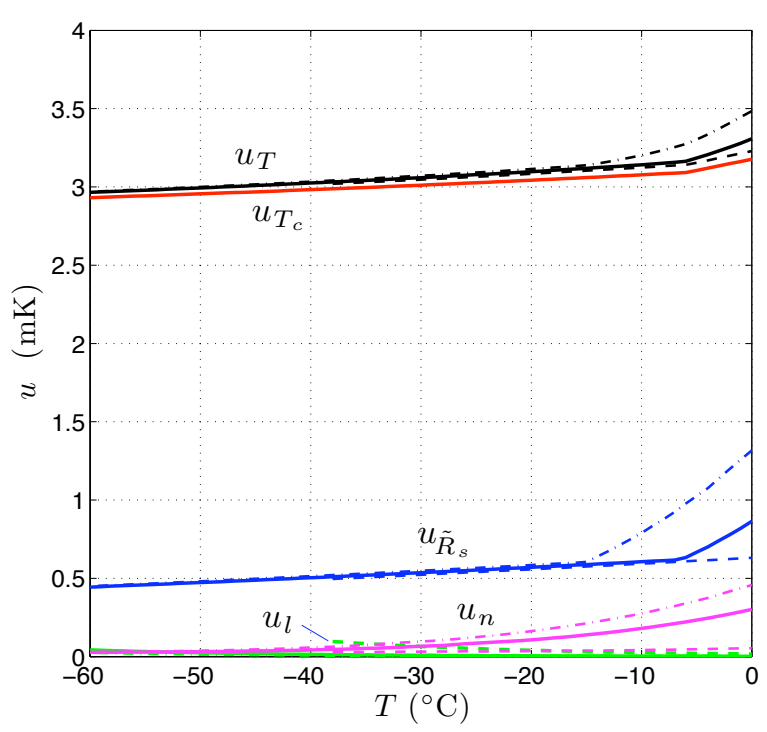

Figure 13. Combined standard uncertainty $u_{T}$ of the ITS-90 temperature measurements obtained with the current PTLS when using the $\mathrm{P}, \mathrm{T} 01$, and T02 probe series (dashed, solid, dash-dot lines, respectively). Also shown are the primary contributors to $u_{T}$, specifically, $u_{T_{c}}$, the standard uncertainty of the temperature-sensor calibrations, and $u_{\tilde{R}_{s}}$, the standard uncertainty of the resistance measurements. The $u_{n}$ curves represent the upper bound for the noise uncertainty under most operating conditions; they assume the hardware noise filters are not used. The standard uncertainty of the resistance corrections is less than 0.1 millikelvin in all situations.

as being due to climate change; to date, borehole paleothermometry generally has been used to analyze the shape of individual temperature profiles. Thus the offset of an entire temperature log due to miscalibration or system drift will not affect a reconstructed climate history and is of little importance in this context. For borehole paleothermometry, the appropriate uncertainties are those that describe the uncertainty of a temperature profile's shape, or expressed another way, the standard uncertainty $\left(u_{T}^{r}\right)$ of the temperature measurements from a single temperature log relative to one another.

An assessment of the temperature uncertainties discussed in Section 3.1 shows that nearly all are associated with errors that potentially can distort a profile's shape and thus should be included in the combined standard uncertainty $u_{T}^{r}$ of relative temperature measurements. However, because the long-term drift of a high-quality instrument will not in general produce an error that distorts a profile, the drift component 


\section{USGS Polar Temperature Logging System}

of a readout's accuracy specification can be dropped while the nonlinearity and internal noise components should be retained. With this change, the standard uncertainty $u_{T}^{r}$ of the relative temperature measurements produced by the PTLS ranges from $1.6 \mathrm{mK}$ at $-60^{\circ} \mathrm{C}$ to $2.0 \mathrm{mK}$ at $0^{\circ} \mathrm{C}$ (fig. 14). The standard uncertainty $u_{T_{c}}^{r}$ of the temperature-sensor calibration (based on instrument specifications) is still the largest contributor, ranging from $1.6 \mathrm{mK}$ at $-60^{\circ} \mathrm{C}$ to $1.8 \mathrm{mK}$ at $0^{\circ} \mathrm{C}$. These uncertainty values are likely to be overestimates because the dominant source of uncertainty affecting $u_{T_{c}}^{r}$ and $u_{T}^{r}$ is the SPRT readout, and it is used only over 1 percent of its range, whereas the nonlinearity specification pertains to the instrument's entire range. Still, without tests to quantify the uncertainty over such a restricted range, we must use the manufacturer's stated full-range uncertainty. At this time we simply note that the residuals from fitting the sensor calibration data to equation (11) typically have standard deviations ranging from 0.20 to $0.45 \mathrm{mK}$, suggesting $u_{T_{c}}^{r}$ (and thus $u_{T}^{r}$ ) may be substantially smaller than the values based on the SPRT readout's full-range nonlinearity specification. To accommodate this possibility, we state that the "upper limit" of $u_{T}^{r}$ ranges from $1.6 \mathrm{mK}$ at $-60^{\circ} \mathrm{C}$ to $2.0 \mathrm{mK}$ at $0^{\circ} \mathrm{C}$. As with $u_{T}$, the uncertainty $u_{T}^{r}$ of relative temperature measurements is classified Type A,B. The $u_{T}^{r}$ upper limit before 2008 was very similar to the current value, ranging from $1.6 \mathrm{mK}$ at $-60^{\circ} \mathrm{C}$ to $2.1 \mathrm{mK}$ at $0^{\circ} \mathrm{C}$.

\subsection{Depth Uncertainties}

It is difficult to accurately determine the depth below surface with any borehole logging system. With the PTLS, systematic depth errors arise from forceinduced strains in the logging cable and winch, tool buoyancy, temperature changes that alter the radius of the measuring wheel, and thermally induced longitudinal strains in the logging cable once it enters the relatively cold borehole. We attempt to correct for these errors (Section 2.3), although there are uncertainties in the corrections. Errors also potentially arise from slippage of the cable on the measuring wheel, debris in the wheel's cable groove, and downhole cable hangups. Cable slippage is minimized by logging at a slow, steady pace $\left(5.5 \mathrm{~cm} \cdot \mathrm{s}^{-1}\right.$, or less). If slippage occurs with the PTLS, some of it presumably is incorporated in the depth-calibration factor $C_{d}$; depth calibration data are collected at the same downward speed and tensions as utilized during a log. The boreholes we $\log$ in polar environments are invariably filled with a

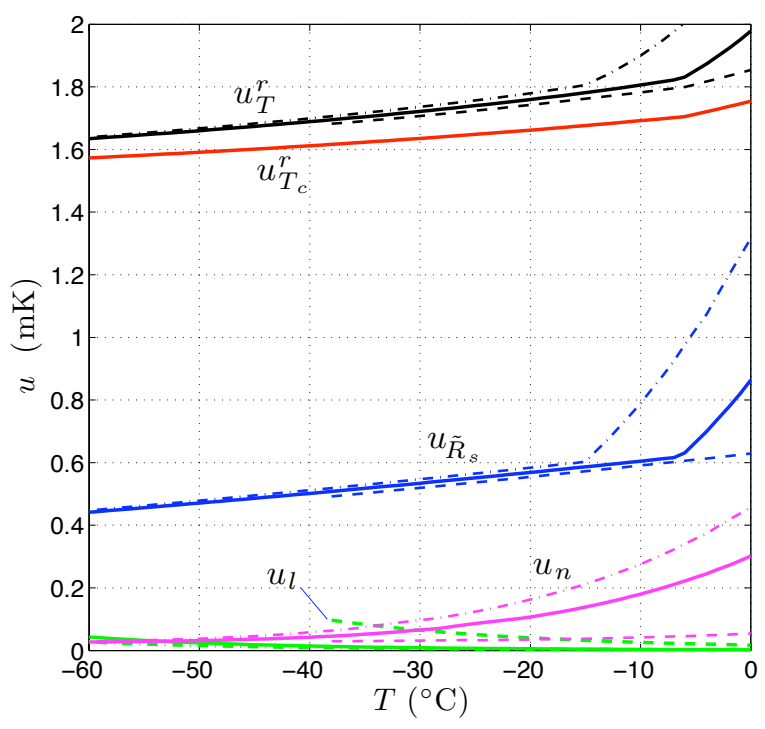

Figure 14. Combined standard uncertainty $u_{T}^{r}$ of relative temperature measurements and the individual uncertainty components. The $u_{T}^{r}$ and $u_{T_{c}}^{r}$ curves represent upper limits. Symbols are identical to those used in figure 13.

clean, nonfreezing fluid (for example, n-butyl acetate, arctic diesel fuel, or a nonaromatic equivalent). As a result, debris in the groove of the measuring wheel that would alter the effective radius of the measuring system has generally not been an issue. Cable and (or) logging-tool hangups within a borehole do sometimes occur. These are detected by monitoring cable tension. When hangups occur, the log is either stopped and restarted once the tool is past the impediment or the logging run is terminated, depending on the severity of the hangup and the objective of the experiment. In the former case, a depth offset with an unknown uncertainty may occur at the obstruction; in the latter case, no depth error arises. When cable hangups do not occur, we are able to quantify the uncertainty $u_{Z}$ of the logging sensor's true depth $Z$ using the root-sumsquare method. Individual uncertainties contributing to $u_{Z}$ are as follows:

Recorded Depth Measurements. The leading term in our estimated true sensor depth (eq. 25) involves the depth recorded by the counter weighted by a calibration factor, $\left[1+C_{d}\left(T_{w}^{\star}, \tilde{Z}^{\star}\right)\right] \tilde{Z}$. Since $C_{d}\left(T_{w}^{\star}, \tilde{Z}^{\star}\right)$ is simply a reference value that is always $\ll 1$, the standard uncertainty of this term is equivalent to the uncertainty $u_{\tilde{Z}}$ of the measurements reported by the depth counter. We establish $u_{\tilde{Z}}$ through statistical 
analysis of multiple determinations of a borehole's total depth according to the counter. Given the PTLS' operating environment, $u_{\tilde{z}}$ is believed to primarily reflect variations in cable slippage on the measuring wheel.

Force-Induced Strains. Starting with equation (19), the standard uncertainty $u_{F}$ of the depth correction for force-induced strains $\left(\delta Z_{F}\right)$ is found to be

$$
u_{F}=\int_{o}^{\tilde{Z}} u_{\mathcal{F}}(\tilde{Z}-\tilde{z}) d \tilde{z}
$$

where $u_{\mathcal{F}}(\tilde{Z})$ is the standard uncertainty of the experimentally determined function $\mathcal{F}(\tilde{Z})$. When $\mathcal{F}(\tilde{Z})$ is adequately represented by a linear function, its depthdependent uncertainty is

$$
u_{\mathcal{F}}(\tilde{Z})=\sigma \sqrt{\frac{1}{n}+\frac{\left(\tilde{Z}-<\tilde{Z}_{i}>\right)^{2}}{\sum_{i=1}^{n}\left(\tilde{Z}_{i}-<\tilde{Z}_{i}>\right)^{2}}} .
$$

Here, $\sigma$ is the standard deviation of the $\mathcal{F}_{i}$ data, $<\tilde{Z}_{i}>$ is the mean value of the calibration depths $\tilde{Z}_{i}$, and $n$ is the number of depth-calibration points (Bowker and Lieberman, 1972). More complicated expressions are required when $\mathcal{F}(\tilde{Z})$ is nonlinear.

Tool Buoyancy. Applying the propagation of uncertainty law to the tool buoyancy correction $\delta Z_{c l F}^{b t}$ (eq. 21), the standard uncertainty $u_{b t}$ of this correction is

$$
\begin{aligned}
u_{b t}=\delta Z_{c l F}^{b t} & {\left[\left(\frac{u_{K}}{K}\right)^{2}+\left(\frac{u_{\Delta m}}{\Delta m}\right)^{2}+\left(\frac{u_{h}}{h+\Delta x}\right)^{2}\right.} \\
+ & \left.\left(\frac{u_{\Delta x}}{h+\Delta x}\right)^{2}\right]^{1 / 2}
\end{aligned}
$$

where $\Delta m=m_{t}^{f}-m_{t}^{a}$. The fractional uncertainty $\left(u_{K} / K\right)$ of the logging cable's elastic stretch coefficient is estimated to be about 0.1 . This greatly exceeds the fractional uncertainties associated with the mass difference $\Delta m$ and the lengths $h$ and $\Delta x$. Thus, uncertainty $u_{b t}$ is well approximated by

$$
u_{b t}=K g\left(m_{t}^{f}-m_{t}^{a}\right)(h+\Delta x)\left(\frac{u_{K}}{K}\right) .
$$

Radial Strain of Measuring Wheel, Thermal. Letting $\phi(t)=T_{w}(t)-T_{w}^{\star}$, the standard uncertainty $u_{w T}$ of the correction for the thermally induced radial strain of the measuring wheel $\left(\delta Z_{w r T}\right.$, eq. 22$)$ during a $\log$ is

$$
\begin{aligned}
u_{w T} & =\left(\frac{R_{w}}{R_{n}}\right)\left[u_{\alpha}^{2}\left(\int_{o}^{\tilde{Z}} \frac{\partial e_{w r T_{w}}}{\partial \alpha} d \tilde{z}\right)^{2}\right. \\
+ & \left.u_{\phi}^{2}\left(\int_{o}^{\tilde{Z}} \frac{\partial e_{w r T_{w}}}{\partial \phi} d \tilde{z}\right)^{2}\right]^{1 / 2}
\end{aligned}
$$

where

$$
\begin{aligned}
& \frac{\partial e_{w r T_{w}}}{\partial \alpha}=\phi(1+3 \alpha \phi)^{-1 / 2}(1+\alpha \phi)^{-3 / 2} \\
& \frac{\partial e_{w r T_{w}}}{\partial \phi}=\alpha(1+3 \alpha \phi)^{-1 / 2}(1+\alpha \phi)^{-3 / 2} .
\end{aligned}
$$

The uncertainty $u_{\alpha}$ of the measuring wheel's thermal expansion coefficient is estimated to be of order $0.1 \alpha$ while uncertainty $u_{\phi}$ is typically $0.1-1.0 \mathrm{~K}$. With these values, $u_{\alpha}$ and $u_{\phi}$ are both significant contributors to $u_{w T}$.

Longitudinal Strain of Logging Cable, Thermal. Uncertainties in the logging cable's thermal expansion coefficient $\alpha$, the temperature difference $\psi=T(z)-T_{w}$, and parameter $f_{T}$ are all significant contributors to the uncertainty $u_{c T}$ of the correction for the thermally induced length change of the cable as it descends a borehole $\left(\delta Z_{c l T}\right.$, eq. 24$)$. Applying the propagation of uncertainty law to $\delta Z_{c l T}$ and dropping negligible terms, $u_{c T}$ is given by

$$
\begin{aligned}
u_{c T}= & {\left[\left(\frac{u_{\alpha}}{\alpha}\right)^{2}\left(\int_{h}^{Z} e_{c l T} d z\right)^{2}+u_{\psi}^{2}[\alpha(Z-h)]^{2}\right.} \\
& \left.+u_{f_{T}}^{2}\left\{\alpha\left[T(h)-T_{w}^{\star}\right] Z\right\}^{2}\right]^{1 / 2}
\end{aligned}
$$

For the logging cable, the fractional uncertainty $\left(u_{\alpha} / \alpha\right)$ is estimated to be $0.1 ; u_{\psi}$ ranges $0.1-1.0 \mathrm{~K}$ while $u_{f_{T}}$ is about 0.1 .

Combined Depth Uncertainty $u_{Z}$. Combining the individual uncertainties, the standard uncertainty of a sensor's true depth is

$$
u_{Z}=\sqrt{u_{\tilde{Z}}^{2}+u_{F}^{2}+u_{b t}^{2}+u_{w T}^{2}+u_{c T}^{2}} .
$$

Ordinarily, $u_{\tilde{Z}}$ and $u_{F}$ are the dominant contributors to $u_{Z}$. Uncertainty components $\left(u_{\tilde{Z}}, u_{F}\right)$ are classified as Type A uncertainties, while $\left(u_{b t}, u_{w T}, u_{c T}\right)$ are Type B. The combined depth uncertainty $u_{Z}$ is thus Type A,B. The magnitude of the individual components and of the combined uncertainty depend to a large degree on specific borehole conditions and the temperature near the winch. 

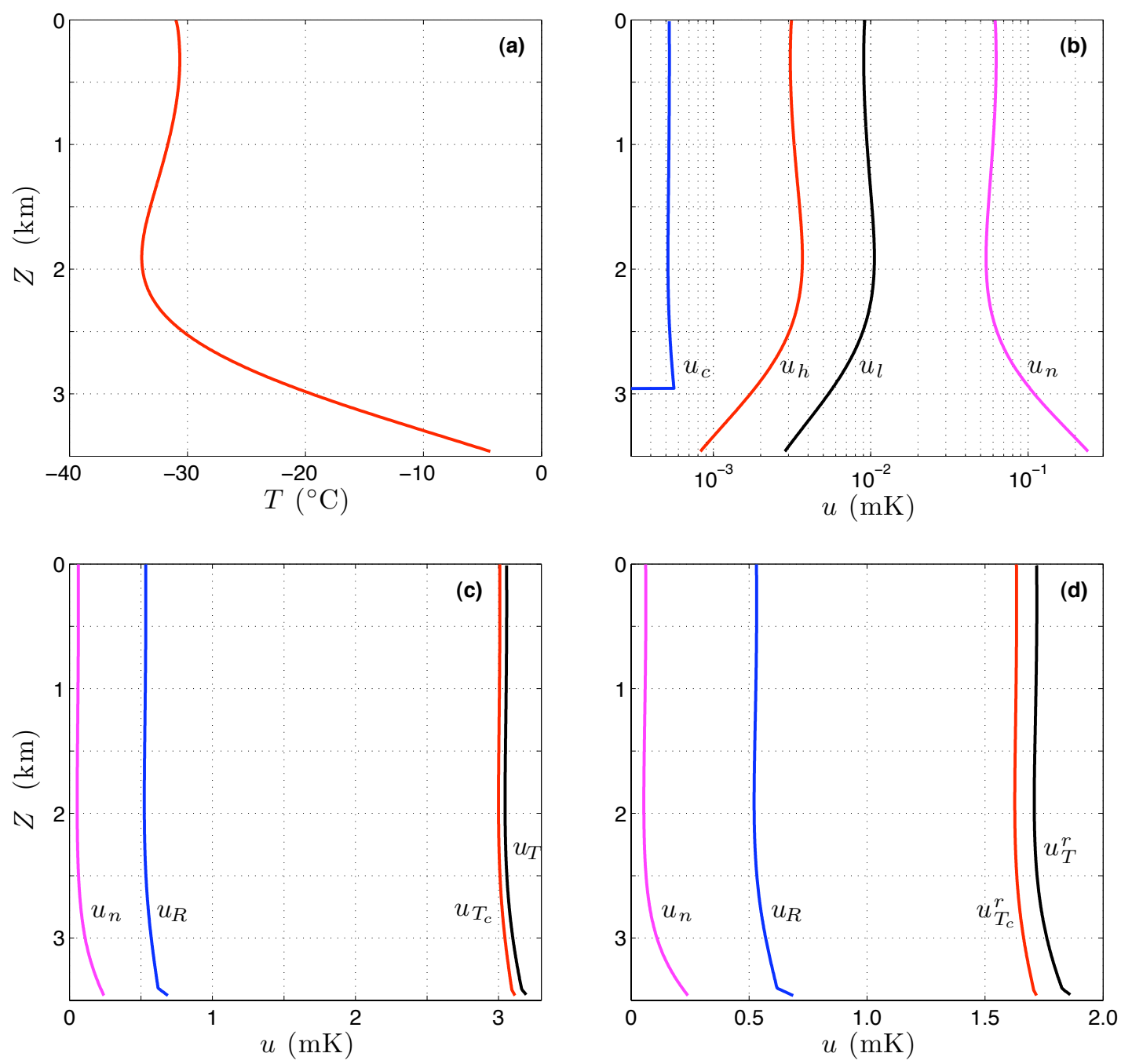

Figure 15. Temperature-measurement uncertainties for the proposed 3.5-kilometer deep WAIS Divide borehole in West Antarctica. (a) Best-guess temperature profile at this site based on ice-flow modeling. (b) Expected resistance-correction uncertainties $\left(u_{l}, u_{h}, u_{c}\right)$ and the noise uncertainty $\left(u_{n}\right)$ assuming the hole is logged with the current PTLS using a T01-series probe at 5.5 centimeters per second. (c) Combined standard uncertainty $\left(u_{T}\right)$ of the ITS-90 temperature measurements along with the largest contributing uncertainties. (d) Combined standard uncertainty $\left(u_{T}^{r}\right)$ of the relative temperature measurements along with the largest contributing uncertainties. The $u_{T}^{r}$ and $u_{T_{c}}^{r}$ curves are upper limits.

\subsection{Example: Proposed WAIS Divide Borehole, Antarctica}

To illustrate the magnitude of the uncertainties and the degree to which they can vary with depth, we consider the conditions at the proposed WAIS Divide Ice Core site in West Antarctica $\left(79^{\circ} 28^{\prime}\right.$ S., $112^{\circ} 05^{\prime}$ W.). Figure 15a shows the "best-guess" temperature profile within the ice sheet at this location based on ice-flow modeling (Tom Neumann, written commun., 2006). We anticipate the fluid in the proposed borehole will be stably stratified (nonconvecting) within 2,000 $\mathrm{m}$ of the surface once the hole is completed. Figure 15b shows the uncertainty of the resistance corrections assuming the hole is logged with the current PTLS using a T01series probe, the logging speed is $5.5 \mathrm{~cm} \cdot \mathrm{s}^{-1}$, a 0.47 $\mu \mathrm{F}$ filtering capacitor is used, and the hardware noise filters are off. With a T01-series probe, the sensor resis- 
tance should range from $20.2 \mathrm{k} \Omega$ at the coldest temperatures encountered $\left(-33.9^{\circ} \mathrm{C}\right)$ to $4.6 \mathrm{k} \Omega$ at the bottom of the hole. The standard uncertainty $\left(u_{l}, u_{h}, u_{c}, u_{e}\right)$ of the resistance corrections are expected to be less than $0.011 \mathrm{mK}$ at all depths while the uncertainty $u_{n}$ associated with electrical noise is predicted to increase from about $0.06 \mathrm{mK}$ in the upper $2,000 \mathrm{~m}$ of the borehole to $0.24 \mathrm{mK}$ at the bottom.

Despite the 30-K temperature change along the profile, the combined standard uncertainty $u_{T}$ of the ITS-90 temperature measurements has only a small depth dependence, varying from $3.05 \mathrm{mK}$ in the upper $2,000 \mathrm{~m}$ to $3.19 \mathrm{mK}$ at the bottom (fig. 15c). This stems from the weak temperature dependence of the dominant term, $u_{T_{c}}$. Similarly, the combined standard uncertainty $u_{T}^{r}$ of the relative temperature measurements that would be used for borehole paleothermometry is only mildly depth-dependent (fig. 15d), ranging from $1.71 \mathrm{mK}$ in the upper $2,000 \mathrm{~m}$ to $1.86 \mathrm{mK}$ at the base of the ice sheet (about 3,465 m).

Figure 16 shows the uncertainty of the temperature sensor's location (depth) when logging the WAIS Divide borehole using plausible values for various parameters. However, it must be emphasized the true depth uncertainties for WAIS Divide temperature logs will depend on the actual borehole conditions and parameter values that occur during those logs. These factors will not be known until the borehole is completed and the hole has been logged several times. For the example shown in figure 16 , we assume the temperature $T_{w}(t)$ of the measuring wheel follows a diurnal cosine-function, fluctuating $\pm 5^{\circ} \mathrm{C}$ about a mean value of $-10^{\circ} \mathrm{C}$, i.e typical summertime temperatures inside the drilling structure where the logging winch will be located. The reference calibration temperature of the wheel $\left(T_{w}^{\star}\right)$ is taken to be $-10^{\circ} \mathrm{C}$, the fluid level in the borehole is set at the firn/ice transition $(h=73 \mathrm{~m})$, parameter $f_{T}$ is 0.3 , and uncertainties $u_{\phi}$ and $u_{\psi}$ are both $1 \mathrm{~K}$. With these values, the standard uncertainty $u_{w T}$ of the depth correction for the thermally induced radial strain in the measuring wheel during a log is limited to 11$12 \mathrm{ppm}$. The correction for the shortening of the cable in the cold borehole has a standard uncertainty $u_{c T}$ ranging from $25 \mathrm{ppm}$ near the surface to a peak value of about $40 \mathrm{ppm}$ when the sensor reaches $Z \approx 1,800 \mathrm{~m}$. For a PTLS temperature sensor, the uncertainty $u_{b t}$ of the tool buoyancy correction is less than $2 \mathrm{ppm}$ and can be ignored.

To account for force-dependent effects on the depth measuring system, we assume in this example that nine pairs of depth-calibration data $(\mathcal{L}, \tilde{\mathcal{L}})$ are col-
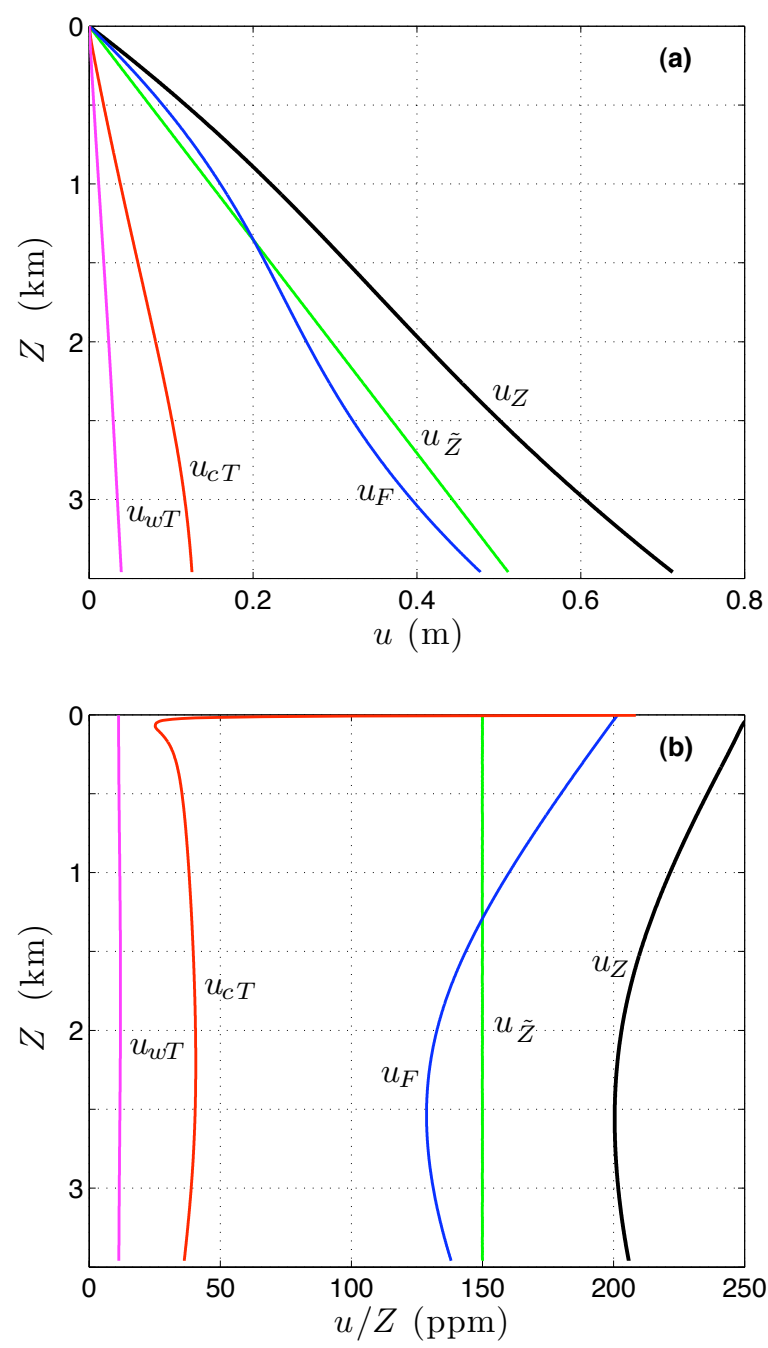

Figure 16. Depth uncertainties for the proposed WAIS Divide borehole as a function of sensor depth $Z$ based on plausible values for various parameters. $\left(u_{F}, u_{c T}, u_{w T}\right)$ are the standard uncertainties of the primary depth corrections while $u_{\tilde{Z}}$ is the standard uncertainty of the measurements reported by the depth counter. The combined standard uncertainty $u_{Z}$ of the sensor's true depth ranges from 200 to 250 parts per million.

lected across the range of borehole depths and that the standard deviation of the fit to the resulting $\mathcal{F}_{i}$ data is $\sigma=3.0 \times 10^{-4}$. This value for $\sigma$ is intermediate between that obtained in the recently logged 1$\mathrm{km}$ Siple Dome A borehole $\left(2.4 \times 10^{-4}\right)$ and the $3-\mathrm{km}$ GISP2-D borehole $\left(4.1 \times 10^{-4}\right)$; GISP2-D was logged before the PTLS winch had a level-wind system, so 
Table 1. Specifications of the USGS Polar Temperature Logging System, mid-2008.

$\left[{ }^{\circ} \mathrm{C}\right.$, degree Celsius; mK, millikelvin; m, meter; cm, centimeter; ppm, parts per million]

\begin{tabular}{l|l}
\hline Temperature range & $-60^{\circ} \mathrm{C}$ to $+23^{\circ} \mathrm{C}$ \\
Temperature resolution & $0.02-0.19 \mathrm{mK}$ \\
Depth range & $0-4,500 \mathrm{~m}$ \\
Depth resolution & $1.0 \mathrm{~cm}$ \\
\hline Standard uncertainty, ITS-90 temperature measurements & $3.0-3.3 \mathrm{mK} \quad$ (Type A,B) \\
Standard uncertainty, relative temperature measurements & $1.6-2.0 \mathrm{mK}^{\dagger} \quad$ (Type A,B) \\
Standard uncertainty, depth measurements & $200-250 \mathrm{ppm}^{\ddagger} \quad$ (Type A,B) \\
\hline$\dagger$ Upper limit. & \\
$\ddagger$ 4.5-km winch with favorable logging conditions. &
\end{tabular}

a much higher value for $\sigma$ is expected from those logs than would occur today. With the proposed set of calibration data, the standard uncertainty $u_{F}$ of the depth correction for force-induced strains ranges from $200 \mathrm{ppm}$ near the surface to a minimum value of $130 \mathrm{ppm}$ at $Z \approx 2,500 \mathrm{~m}$. The greatest unknown in the projected depth uncertainties for the WAIS Divide borehole involves the standard uncertainty $u_{\tilde{Z}}$ of the measurements reported by the depth counter. For the final set of logs obtained in the Siple Dome A borehole, the uncertainty $u_{\tilde{Z}}$ was about $140 \mathrm{ppm}$. Thus, when logging conditions are favorable, the repeatability of the measurements reported by the depth counter is fairly high. In the WAIS Divide example, we use a nominal value for $u_{\tilde{Z}}$ of $150 \mathrm{ppm}$. Combining the individual uncertainty terms, the standard uncertainty of the temperature sensor's true depth ranges from 200 to $250 \mathrm{ppm}$ (fig. 16b).

\section{Summary}

From its origins in the early 1990s, the USGS Polar Temperature Logging System has evolved into a reliable high-precision data-acquisition system for cold polar environments. This field-proven system has been extensively used in Greenland, Antarctica, and arctic Alaska. With a temperature resolution better than $0.2 \mathrm{mK}$, the PTLS is capable of detecting small subsurface temperature changes due to fluid convection and other phenomena. Our initial goal of reducing the uncertainty of the temperature measurements to about
$1 \mathrm{mK}$ has proven difficult to achieve. The standard uncertainty $u_{T}$ of the system's ITS-90 temperature measurements is $3.0-3.3 \mathrm{mK}$. This is more than adequate for climate-change detection and monitoring, especially in the Arctic where contemporary surface-temperature changes exceeding $1 \mathrm{~K} /$ decade have recently been observed. Relative temperature measurements used to reconstruct past climate changes with borehole paleothermometry have a standard uncertainty $u_{T}^{r}$ whose upper limit ranges from 1.6 to $2.0 \mathrm{mK}$. This is tantalizingly close to $1 \mathrm{mK}$.

The uncertainty of the temperature sensor's location (depth) during a log depends on specific borehole conditions and the temperature near the measuring wheel. Thus the depth uncertainty must be treated on a case-by-case basis. However, recent experience with our large winch indicates that when conditions are favorable (that is the winch is operated within a shelter, steady power is available for the winch motor, fluid in the borehole is free of debris, and so forth), the $4.5-\mathrm{km}$ system can produce depths with a standard uncertainty $u_{Z}$ on the order of $200-250 \mathrm{ppm}$. The small helicopter-transportable winches have undergone a number of design changes recently. Although the depth-measurement system for the small winches is very similar to the $4.5-\mathrm{km}$ system, we do not yet have enough information about various parameters to quantitatively assess $u_{Z}$ for logs acquired with these portable winches. The current specifications for the USGS Polar Temperature Logging System are summarized in Table 1. 


\section{Appendix: Nomenclature}

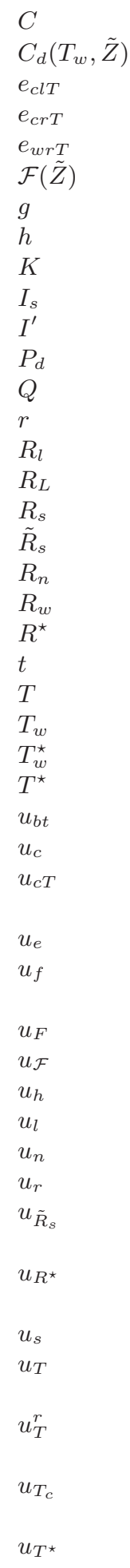

capacitance

depth calibration function

strain, cable, radial, thermal

force-dependent function

gravitational acceleration

elastic stretch coefficient

source current

extraneous current (noise)

Seebeck coefficient

radius, logging cable

lead resistance (Kelvin circuit)

radius, depth-measuring wheel

time

temperature

SPRT reference temperature

readout measurements

temperature measurements

temperature measurements

-sensor calibration strain, cable, longitudinal, thermal

strain, measuring wheel, radial, thermal

depth to air/fluid interface in a borehole

power-dissipation constant, thermistor

interconductor leakage resistance

temperature-sensor resistance (true)

temperature-sensor resistance (measured)

nominal radius used by depth counter

sensor resistance during calibration

measuring wheel temperature

reference depth-calibration temperature

standard uncertainty, tool buoyancy correction

standard uncertainty, capacitance correction

standard uncertainty, correction for thermal

strain (longitudinal) of logging cable

standard uncertainty, thermal EMFs

standard uncertainty of temperature-sensor

calibration due to bath fluctuations

standard uncertainty, force-induced corrections

standard uncertainty of function $\mathcal{F}$

standard uncertainty, self-heating correction

standard uncertainty, leakage correction

standard uncertainty, instrumental noise

standard uncertainty, resistance readout

combined standard uncertainty, resistance

standard uncertainty, temperature-sensor

resistance measurements during calibration

standard uncertainty, resistance standards

combined standard uncertainty, ITS-90

combined standard uncertainty, relative

combined standard uncertainty, temperature

combined standard uncertainty, SPRT reference temperature

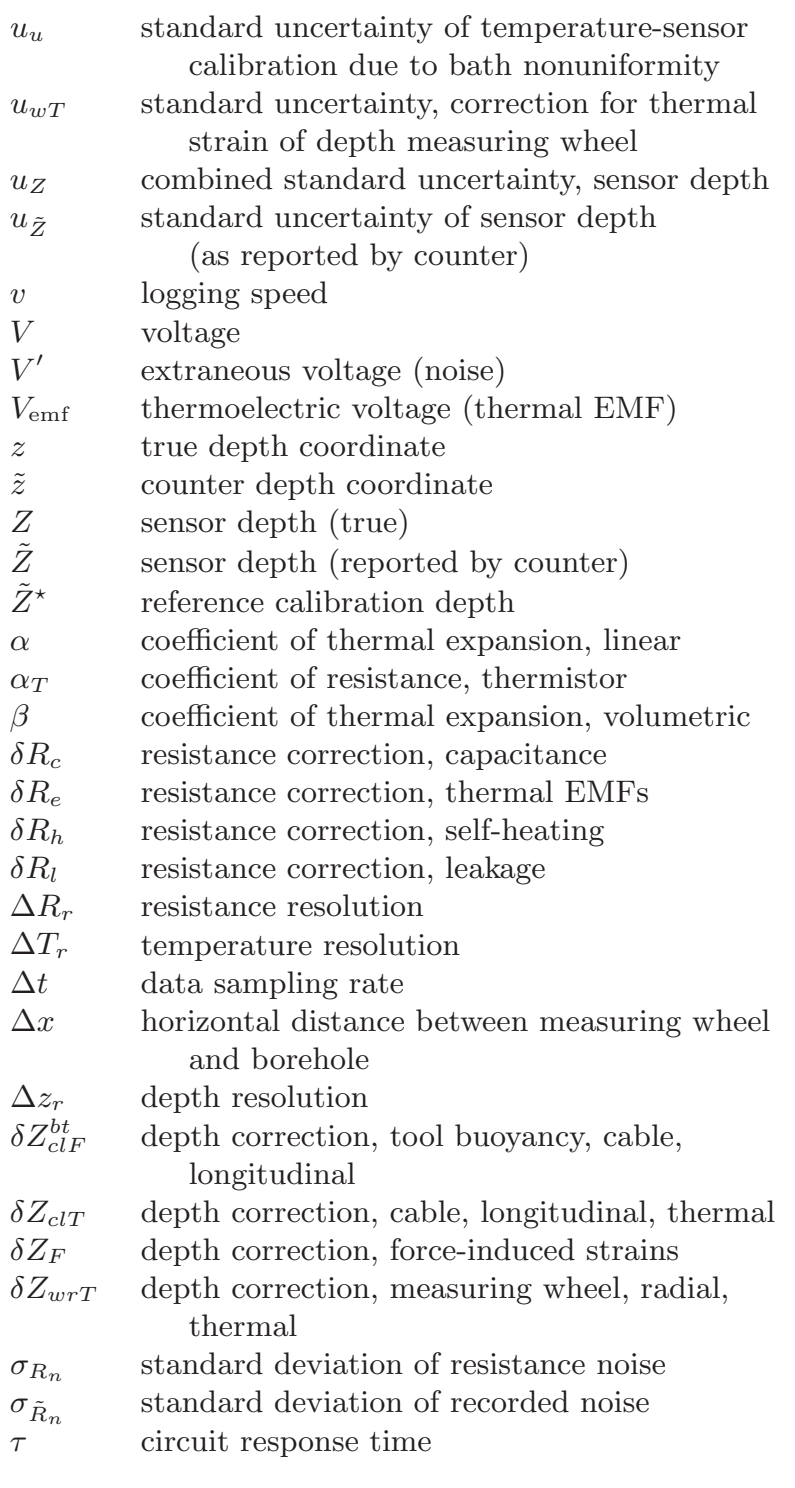

Acknowledgments. I gratefully thank Jack Kennelly and Mark Ohms for their contributions to the design of the PTLS and their exceptional skill in fabricating it. I also thank Frank Urban for performing experiments used to quantify some of the critical measurement uncertainties, Tom Neumann for supplying the "best-guess" temperature profile for the proposed WAIS Divide Ice Core site (West Antarctica), and the reviewers for their helpful comments. Funding for this work was provided by the U.S. Geological Survey's Earth Surface Dynamics Program. 


\section{References}

Bevington, P.R., 1969, Data reduction and error analysis for the physical sciences: New York, McGraw-Hill, $336 \mathrm{p}$.

Bowker, A.H., and Lieberman, G.J., 1972, Engineering statistics (2d ed.): Englewood Cliffs, N.J., PrenticeHall, $641 \mathrm{p}$.

Clow, G.D., 1992, The extent of temporal smearing in surface-temperature histories derived from borehole temperature measurements: Palaeogeography, Palaeoclimatology, Palaeoecology, v. 98, p. 81-86.

Clow, G.D., Saltus, R.W., and Waddington, E.D., 1996, A new high-precision borehole temperature logging system used at GISP2, Greenland and Taylor Dome, Antarctica: Journal of Glaciology, v. 42, p. 576-584.

ISO, 1993a, International vocabulary of basic and general terms in metrology (2d ed.): International Organization for Standardization, Geneva, Switzerland, 59 p. (ISBN 92-67-01075-1)

ISO, 1993b, Guide to the expression of uncertainty in measurement: International Organization for Standardization, Geneva, Switzerland, 110 p. (ISBN 92-67-10188-9)

Mangum, B.W., and Furukawa, G.T., 1990, Guidelines for realizing the International Temperature Scale of 1990 (ITS-90): NIST Technical Note 1265, National Institute of Standards and Technology, $176 \mathrm{p}$.

McGee, T.D., 1988, Principles and methods of temperature measurement: New York, Wiley, 581 p.

Misiti, M., Misiti, Y., Oppenheim, G., and Poggi, J., 2005, Wavelet Toolbox User's Guide, Fourth printing: The MathWorks Inc., 1034 p.

Parker, R.L., 1994, Geophysical inverse theory: Princeton, N.J., Princeton University Press, 386 p.

Saltus, R.W., and Clow, G.D., 1994, Deconvolution of continuous borehole temperature logs: examples from the Greenland GISP2 icecore hole: U.S. Geological Survey Open-File Report 94-254, 42 p.

Sass, J.H., Lachenbruch, A.H., Munroe, R.J., Greene, G.W., and Moses, Jr., T.H., 1971, Heat flow in the western United States: Journal of Geophysical Research, v. 15, p. 6376-6413.

Steinhart, J.S., and Hart, S.R., 1968, Calibration curves for thermistors: Deep-Sea Research, v. 15, p. 497-503.

Taylor, B.N., and Kuyatt, C.E., 1994, Guidelines for evaluating and expressing the uncertainty of NIST measurement results: NIST Technical Note 1297, National Institute of Standards and Technology, $20 \mathrm{p}$. 
Publishing support provided by:

Denver Publishing Service Center

Manuscript approved for publication, July 2008

Edited by Mary A. Kidd

Layout by Gary D. Clow

For more information concerning this publication, contact:

Science Center Chief, USGS Rocky Mountain Geographic Science Center

Box 25046, Mail Stop 516

Denver, CO 80225

(303) 202-4106

Or visit the Earth Surface Processes Team Web site at:

http://esp.cr.usgs.gov/ 